Article

\title{
Zika Virus Infection of Sertoli Cells Alters Protein Expression Involved in Activated Immune and Antiviral Response Pathways, Carbohydrate Metabolism and Cardiovascular Disease
}

\author{
Mahamud-ur Rashid ${ }^{1,2, *}$, Ying Lao ${ }^{2}$, Victor Spicer ${ }^{2}$ and Kevin M. Coombs ${ }^{1,2,3}$ \\ 1 Department of Medical Microbiology and Infectious Diseases, The University of Manitoba, Room 543 Basic \\ Medical Sciences Building, 745 Bannatyne Avenue, Winnipeg, MB R3E 0J9, Canada; \\ kevin.coombs@umanitoba.ca \\ 2 Manitoba Centre for Proteomics \& Systems Biology, Room 799, 715 McDermot Avenue, \\ Winnipeg, MB R3E 3P4, Canada; ying.lao@umanitoba.ca (Y.L.); victor.spicer@umanitoba.ca (V.S.) \\ 3 Children's Hospital Research Institute of Manitoba, Room 513, John Buhler Research Centre, \\ 715 McDermot Avenue, Winnipeg, MB R3E 3P4, Canada \\ * Correspondence: rashidmm@myumanitoba.ca
}

check for updates

Citation: Rashid, M.-u.; Lao, Y.; Spicer, V.; Coombs, K.M. Zika Virus Infection of Sertoli Cells Alters Protein Expression Involved in Activated Immune and Antiviral Response Pathways, Carbohydrate Metabolism and Cardiovascular Disease. Viruses 2022, 14, 377. https://doi.org/10.3390/v14020377

Academic Editor: Subir Sarker

Received: 11 January 2022

Accepted: 7 February 2022

Published: 11 February 2022

Publisher's Note: MDPI stays neutral with regard to jurisdictional claims in published maps and institutional affiliations.

Copyright: (C) 2022 by the authors. Licensee MDPI, Basel, Switzerland. This article is an open access article distributed under the terms and conditions of the Creative Commons Attribution (CC BY) license (https:// creativecommons.org/licenses/by/ $4.0 /)$.

\begin{abstract}
Zika virus (ZIKV), a re-emerging virus, causes congenital brain abnormalities and GuillainBarré syndrome. It is mainly transmitted by Aedes mosquitoes, but infections are also linked to sexual transmissions. Infectious ZIKV has been isolated, and viral RNA has been detected in semen over a year after the onset of initial symptoms, but the mode of long-term persistence is not yet understood. ZIKV can proliferate in human Sertoli cells (HSerC) for several weeks in vitro, suggesting that it might be a reservoir for persistent ZIKV infection. This study determined proteomic changes in HSerC during ZIKV infections by TMT-mass spectrometry analysis. Levels of 4416 unique Sertoli cell proteins were significantly altered at 3, 5, and 7 days after ZIKV infection. The significantly altered proteins include enzymes, transcription regulators, transporters, kinases, peptidases, transmembrane receptors, cytokines, ion channels, and growth factors. Many of these proteins are involved in pathways associated with antiviral response, antigen presentation, and immune cell activation. Several immune response pathway proteins were significantly activated during infection, e.g., interferon signaling, T cell receptor signaling, IL-8 signaling, and Th1 signaling. The altered protein levels were linked to predicted activation of immune response in HSerC, which was predicted to suppress ZIKV infection. ZIKV infection also affected the levels of critical regulators of gluconeogenesis and glycolysis pathways such as phosphoglycerate mutase, phosphoglycerate kinase, and enolase. Interestingly, many significantly altered proteins were associated with cardiac hypertrophy, which may induce heart failure in infected patients. In summary, our research contributes to a better understanding of ZIKV replication dynamics and infection in Sertoli cells.
\end{abstract}

Keywords: Zika virus; Sertoli cells; sexual transmission; persistence in semen; immune response; glycolysis; carbohydrate metabolism; cardiovascular disease

\section{Introduction}

Zika virus (ZIKV) belongs to the family Flaviviridae, and is a single-stranded positivesense RNA virus. Other members of the family include Japanese encephalitis virus (JEV), Yellow fever virus (YFV), West Nile virus (WNV) and Dengue virus (DENV). ZIKV was first discovered in Rhesus monkeys in Uganda's Zika rainforest in 1947 [1].

However, the virus remained undiagnosed for a long time due to the disease's nonspecific flu-like symptoms and a lack of diagnostic screening [2,3]. In 2007, ZIKV re-emerged in the Pacific islands, spreading to over 80 countries/territories, including Latin America, the United States, and Southeast Asia [4-8]. The virus has been linked to microcephaly 
in babies [9] and Guillain-Barré syndrome (GBS) in adults [10,11]. Therefore, the World Health Organization (WHO) considers ZIKV to be a threat to global health, and declared a global health emergency in 2016 [12].

In endemic regions, ZIKV is mainly transmitted by mosquito bites (Aedes aegypti and A. albopictus) [13]. However, the virus can persist in the male reproductive tract and contribute to sexual transmission [14-16]. After the initial onset of symptoms, ZIKV RNA and infectious virus particles were detected in semen from infected males after 414 days [17] and 69 days [18], respectively. Sexual transmission of ZIKV has been reported in 14 countries so far [19]. ZIKV was found in murine testis and monkey models after inoculation, causing severe testicular damage [20-23]. Unlike the animal models, ZIKV infection does not cause any noticeable impact on human testis morphology. However, persistent ZIKV infection in the male genital tract may impact testicular hormone levels and sperm quality [24-26]. These findings indicate that, like many other viruses [27], ZIKV exploits the immune-privileged environment of the testis, which lacks an adaptive immune system [28], and hides for a prolonged time. However, the cellular reservoir of ZIKV testicular persistence is not clearly understood.

Sertoli, Leydig, and germ cells are the most prevalent cells found in human testes [29]. Sertoli cells constitute the major part of the testicular environment and play a critical role in the development of spermatogonial stem cells into mature sperm [30,31]. The testis-blood barrier, which protects the male reproductive system from pathogens, is also established by Sertoli cells [32]. Another major cell type in the testes is the Leydig cell, which secretes the male sex hormone testosterone and is essential for the development of male reproductive organs and characteristics [33]. In mouse testis, Sertoli cells are the most susceptible to ZIKV infection [34]. A previous study has demonstrated that ZIKV can cross the bloodtestis barrier and efficiently infect Sertoli cells [35]. Moreover, the virus not only can infect Sertoli cells, but can persist for several days/weeks without any observable cytopathic impact $[34,36,37]$.

We previously used an aptamer-based assay and demonstrated that ZIKV infection caused alterations in the levels of proteins involved in spermatogenesis in HSerC [36]. In order to better understand the mechanism(s) of viral persistence and its impact on the male genital tract, we extended the previous study by using a complementary tandem mass tag (TMT)-based 2D LC/MS/MS mass spectrometry-based approach to investigate Sertoli cell proteomic alterations after ZIKV infection. We measured $\sim 8000$ proteins across three time points and identified approximately 4400 Sertoli cell proteins significantly affected by ZIKV. The possible significance of these proteins in cellular processes, signaling pathways, and disease pathogenesis were investigated through bioinformatics analyses.

\section{Materials and Methods}

\subsection{Cells}

Primary Sertoli cells (HSerC) isolated from human testis were purchased from ScienCell Research Laboratories, CA, USA (Catalog \#4520). The cells were cultured at $37{ }^{\circ} \mathrm{C}$ in $5 \%$ $\mathrm{CO}_{2}$ in poly-L-lysine-(Sigma-Aldrich, ON, Canada, Cat.\# P4707) coated culture plates in Sertoli Cell Medium (ScienCell Research Laboratories, CA, USA, Cat. \#4521). Following the company's recommendation, Sertoli cells were trypsinized to detach them from the culture surface and passaged every 2-3 days for maintenance. All experiments were conducted using the cells that had reached passage 7 .

\subsection{Virus}

The Zika virus strain used in this study (ZIKV/Homo sapiens/PRI/PRVABC59/2015) was donated by Dr. David Safronetz, Chief of Special Pathogens, National Microbiology Laboratory, Public Health Agency of Canada. For future use, the ZIKV strain was expanded in Vero cells (ATCC, Manassas, VA, USA, Cat. \#CCL-81) and kept at $-80{ }^{\circ} \mathrm{C}$ in $10 \%$ FBS (Thermo Fisher Scientific, Waltham, CA, USA, Cat \#A4766801). The stock virus was titered in Vero cells by plaque assay. 


\subsection{Infection}

HSerC were grown in $75 \mathrm{~cm}^{2}$ cell culture flasks treated with poly-L-lysine. At $70 \%$ confluency, cells were infected with ZIKV at a multiplicity of infection (MOI) of 3 plaqueforming units (PFU) per cell. Culture plates were rocked every $10-15 \mathrm{~min}$ for $2 \mathrm{~h}$ in a $37^{\circ} \mathrm{C}$ incubator (Thermo electron corporation, Waltham, MA, USA) to allow the virus to attach to cell surfaces, and cells were overlaid with Sertoli cell media containing $2.5 \%$ FBS. At 3, 5 , and 7 days after infection, ZIKV-infected and mock, non-infected cells were collected. All experiments were done in three biological replicates.

\subsection{Protein Extraction and Quantification}

The Sertoli cells infected with ZIKV and time-matched mock-treated cells were scraped from the culture plates after 3,5 and 7 days of infection. Centrifugation at $600 \times g$ for 8 min pelleted the cells, which were then washed three times with sterile ice-cold PBS(Thermo Fisher Scientific, Waltham, CA, USA). The pelleted cells were then lysed in 4\% SDS in $100 \mathrm{mM}$ HEPES buffer $\mathrm{pH} 8.5$ by sonication. Centrifugation at $14,000 \times \mathrm{g}$ for $10 \mathrm{~min}$ at $4{ }^{\circ} \mathrm{C}$ was used to remove insoluble cellular components. Bradford Protein Assay was used to determine the protein concentrations in the supernatants (Bio-Rad, Hercules, CA, USA, Cat. 5000001).

\subsection{Immunoblotting}

The protein concentrations in the cell lysates were measured using the Bradford Protein Assay, and $20 \mu \mathrm{g}$ of protein were resolved in 10\% SDS-PAGE gels and transferred to $0.2 \mu \mathrm{m}$ nitrocellulose membranes. Anti-PSMA2 (Cell Signaling, Danvers, MA, USA, Cat. 2455), anti-ZIKV NS1 (BioFront Technologies, Tallahassee, FL, USA, Cat. BF-1225-06), anti-ZIKV NS3 (Genetex, Irvine, CA, USA, Cat No. GTX133309), anti-ZIKV Env (Genetex, Cat No. GTX133314), anti-STAT1 (Cell Signaling, Cat. 9176S), and anti-Beta-Actin (Cell Signaling, Cat. 3700S) antibodies were used to detect protein targets. Anti-rabbit (Cell Signaling, Cat. \#7074) or anti-mouse (Cell Signaling, Cat. \#7076) secondary antibody was used to identify the primary antibody conjugates to the targeted proteins. After overlaying with ECL reagents, protein bands were photographed with an Amersham Imager 680 (Gelifesciences, MA, USA). To quantify band intensities, Image J version. 1.53e (NIH, Bethesda, MD, USA) was used, and Graphpad Prism version 6.0. (La Jolla, CA, USA) was used to visualize them graphically.

\subsection{Tandem Mass Tags (TMT) Mass Spectrometry Analyses and Protein Quantification}

To determine the impact of ZIKV infection on the cellular proteome, a total of 18 protein samples were collected from ZIKV- and mock-infected Sertoli cells at 3, 5 and 7 days post-infection (dpi). Proteins were digested into peptides by the SP3 (single-pot solidphase-enhanced sample preparation) methods as described elsewhere [38,39]. In summary, peptides were eluted after digestion of the proteins with trypsin for $14 \mathrm{~h}$ at $37^{\circ} \mathrm{C}$. Sixplex TMT labeling was performed for mock and infected samples of the same time points following the manufacturer's (Thermo Fisher Scientific, Waltham, CA, USA) instructions. An equal amount of six TMT labeled samples were mixed together and 2D LC/MS/MS was performed using an Orbitrap Q Exactive HF-X instrument [40] (Thermo Fisher Scientific, Bremen, Germany). For identification of the peptide/proteins, ZIKV (Thai strain) and human (Uniprot 2016) databases were used as references. The intensities of TMT6 peptide level reporter tags were averaged over a $\pm 0.1 \mathrm{Da}$ window and corrected for isotopic overlap between channels using the batch-specific correction matrix provided. The sum of peptide level TMT6 reporter tag intensities for each protein was transformed into a $\log _{2}$ scale for easier differential analysis. 


\subsection{Statistical and Bioinformatics Analyses}

Initially, alterations in the levels of any individual protein expression were determined by the differences between $\log _{2}$ values (Delta $\log$ ) of an infected and time-matched mock sample. Then, the delta $\log _{2}$ values were converted to fold-change for each of the proteins. The $p$-value was determined by the Students $t$-test ( 2 tails) and Z-score analyses based on the protein expression difference of all three replicates. $p$-value $<0.05$ and Z-score values of $\geq .96 \sigma$ and $\leq-1.96 \sigma$ were considered significant as described before [41]. The lists of significantly altered proteins were uploaded into Ingenuity Pathway Analysis (IPA) software, and core analysis was done with a cut-off value $p$-value $<0.05$ and fold change above 1.5 or below -1.5 . The IPA core analysis predicted the top affected canonical pathways, bio-functions, interconnecting networks and upstream molecules based on these protein level changes. The Western blot image band intensities were quantified using Image J version1.53e software (NIH, Bethesda, MD, USA), and statistical analyses were performed by one-way or two-way ANOVA ( $p$-values $<0.05$ ) in GraphPad Prism version 6.0. MORPHEUS (Broad Institute, Cambridge, MA, USA), a free internet software program, was used to generate the heatmaps.

\section{Results}

\subsection{Infectivity of ZIKV and Its Cytopathic Effect in Primary HSerC}

After infecting HSerC with ZIKV at MOI = 3, viral protein expression levels, which could result from increased expression, lower protein turnover, or a combination of both, and cytopathic effects were monitored at 3, 5 and $7 \mathrm{dpi}$, ZIKV infection did not induce any cytopathic effects (Figure 1A). However, three viral proteins (NS1, NS3, and E) were expressed at all time points in the infected cells, with maximal levels at $3 \mathrm{dpi}$; by 5 and $7 \mathrm{dpi}$, their expressions had dropped significantly (Figure 1B,C). Mass spectrometry analysis of Zika viral protein levels revealed that most of the proteins were at their highest levels at $3 \mathrm{dpi}$, with the exception of NS2B, NS4A, and C, which peaked at $5 \mathrm{dpi}$. All ZIKV proteins were expressed to the lowest level at $7 \mathrm{dpi}$ (Figure 1D). In our previous study, we did not observe any significant protein level alterations at the early stage (1 dpi) of replication. However, virus titer peaks at $5 \mathrm{dpi}$ and declines at $7 \mathrm{dpi}$ [36]. Therefore, we selected day 3 (mid), day 5 (peak viral titer) and day 7 (late stage) for subsequent proteomic analyses based on these observations.

\subsection{The Impact of ZIKV Infection on the HSerC Cellular Proteome}

TMT-based mass spectrometry was used to determine the proteomic alterations in protein levels caused by ZIKV infection in HSerC, which detected more than 6000 proteins from each sample (Figure 2A). Among them, 4423 unique protein levels were significantly affected considering all three time points. A total of 2367 (853 up-regulated and 1514 downregulated), 2363 (1651 up-regulated and 712 down-regulated), and 1782 (1209 up-regulated and 573 down-regulated) proteins were significantly ( $p$ value $<0.05$ ) affected at 3,5 and 7 dpi, respectively (Table 1). 
A
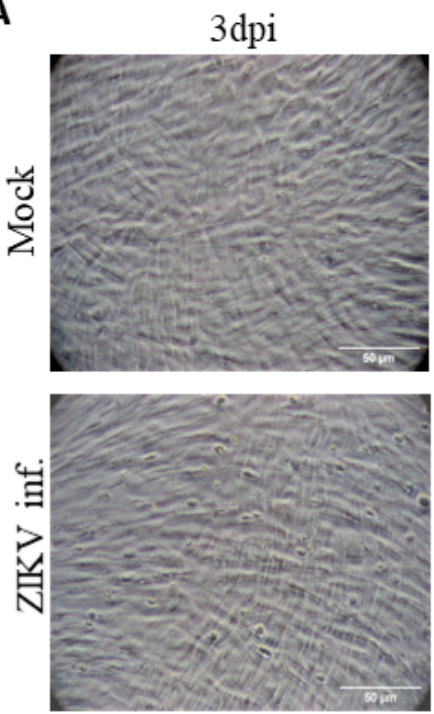

B

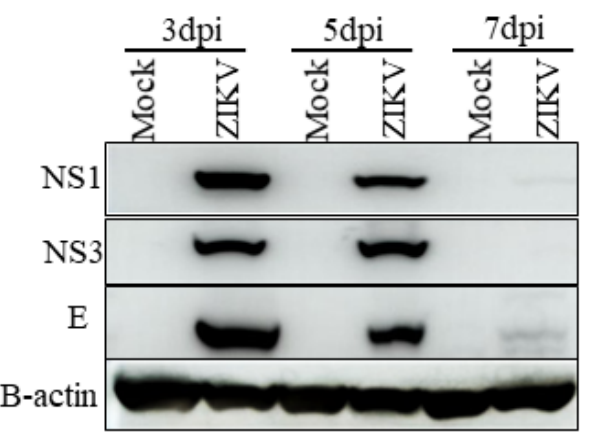

5 dpi
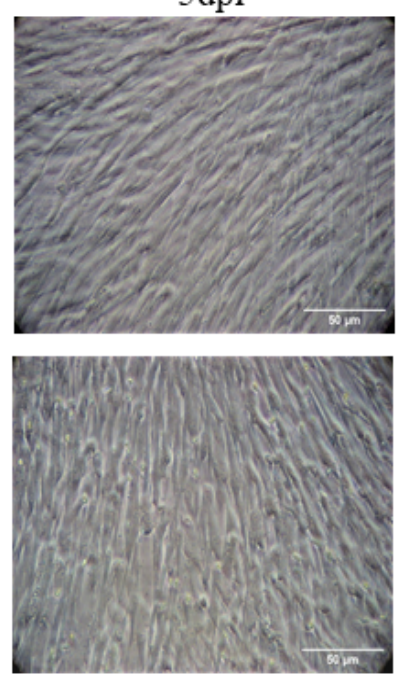

C
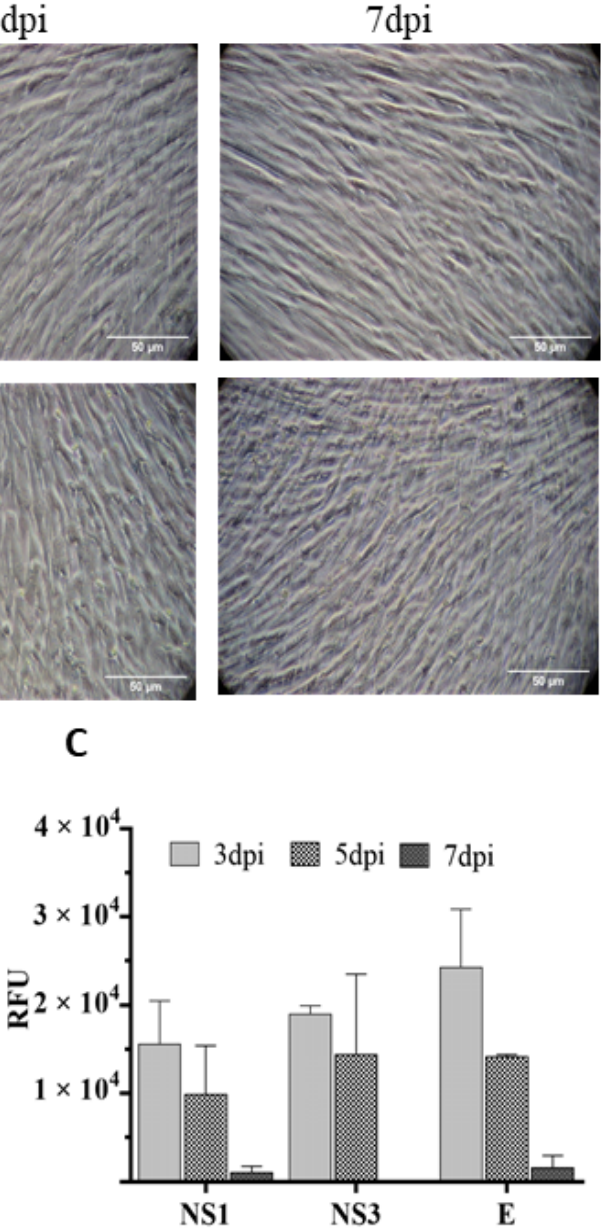

D

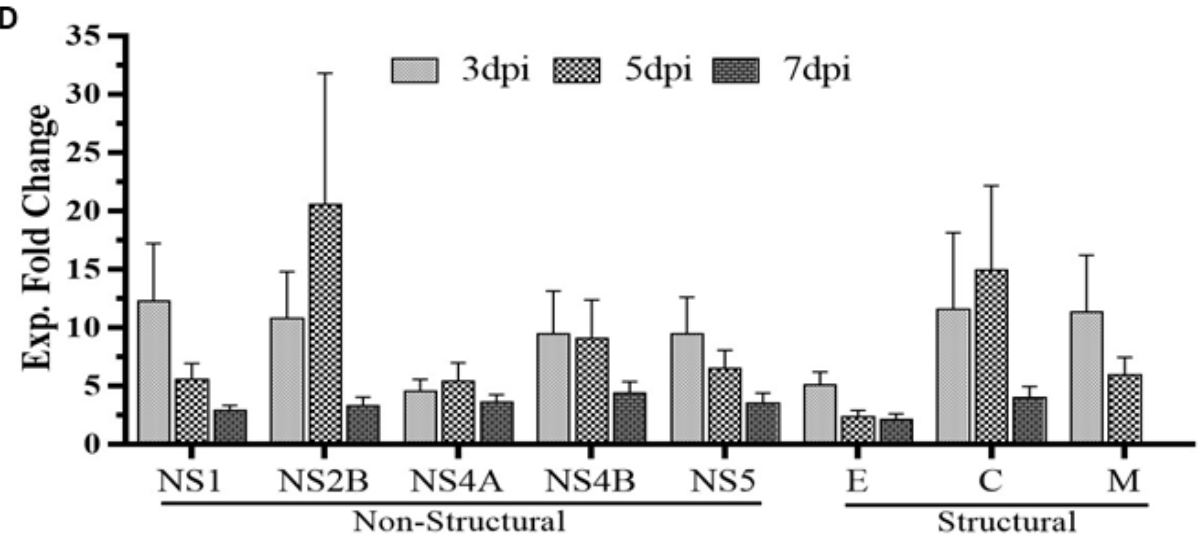

Figure 1. Cytopathic impact of ZIKV infection on HSerC and viral protein expression. HSerC were infected with ZIKV an MOI of 3. (A) Cytopathic impact of ZIKV infection was observed at 3, 5 and 7 days post infection (dpi) under bright-field microscopy at $200 \times$ magnification. Scale bar is $50 \mu \mathrm{m}$. (B) Viral protein expression was determined by Western blot using ZIKV-NS1, NS3 and ENV monoclonal antibodies at 3, 5 and 7 dpi. (C) Quantitative expression of ZIKV viral proteins determined by densitometry analysis of Western blot images using Image J and normalized to B-actin expression. (D) ZIKV proteins expression detected after 3, 5 and 7 dpi by mass spectrometry. Log2 expressions of ZIKV proteins were compared with mock-treated cells and converted to Fold Change (FC) Abbreviations. dpi $=$ Days post infection. Exp $=$ Expression. RFU $=$ Relative fluorescence units. 
Heatmaps of the most affected proteins (Fold change $\geq 2.5$ or $\leq-2.5 ; p$-value $<0.05$ ) revealed that certain protein levels were affected at specific time periods while other proteins were significantly affected at all times (Figure 2B). The topmost up-regulated proteins were Regulator of G-protein signaling 5 (RGS5, Fold change (FC): 45.4), F-box only protein 11 (FBXO11, FC: 41.1), E3 ubiquitin-protein ligase UHRF2 (UHRF2, FC: 17.5), Tyrosinase (TYR, FC: 15.6), Ankyrin-3 (ANK3, FC: 13.2) and the down-regulated were Forkhead box protein Q1 (FOXQ1, FC: -4.74), Kinesin-like protein (KIF1A, FC: -4.37), Collagen alpha-1(I) chain (COL1A1, FC: -3.95), Protein cramped-like (CRAMP1, FC: -4.02), and Hornerin (HRNR, FC: -3.93) (Figure 2B, Table 2). Principal component analysis (PCA) also showed that the protein samples were clustered by time point (Figure 2C).

The volcano plot analysis of the proteins revealed that the majority of the highly affected proteins were down-regulated at $3 \mathrm{dpi}$ (Figure 2D). In contrast, the most significantly up-regulated were at 5 and $7 \mathrm{dpi}$ (Figure 2E,F). The altered proteins belong to different classes of proteins, including enzymes, transcription regulators, kinases, transporters, peptides, cytokines, transmembrane receptors, phosphatases, G protein-coupled receptors, growth factors, ion channels, transcription regulators and others (Figure 2G). We applied $+/-1.5$-fold fold-change cut-off with $p<0.05$ for subsequent more complete bioinformatics analysis.

Table 1. Numbers of significantly affected ZIKV-infected Sertoli proteins.

\begin{tabular}{|c|c|c|c|c|}
\hline $\begin{array}{l}\text { Number That } \\
\text { Are Significant }\end{array}$ & Total Unique & 3 dpi & $5 \mathrm{dpi}$ & $7 \mathrm{dpi}$ \\
\hline and F.C. $>1.00$ & \multirow{2}{*}{4423} & 853 & 1651 & 1209 \\
\hline and F.C. $<1.00$ & & 1514 & 712 & 573 \\
\hline and F.C. $>1.10$ & \multirow{2}{*}{4361} & 832 & 1632 & 1197 \\
\hline and F.C. $<-1.10$ & & 1495 & 700 & 559 \\
\hline and F.C. $>1.25$ & \multirow{2}{*}{2234} & 393 & 744 & 647 \\
\hline and F.C. $<-1.25$ & & 755 & 329 & 193 \\
\hline and F.C. $>1.33$ & \multirow{2}{*}{1342} & 286 & 468 & 382 \\
\hline and F.C. $<-1.33$ & & 395 & 197 & 90 \\
\hline and F.C. > 1.50 & \multirow{2}{*}{619} & 180 & 246 & 176 \\
\hline and F.C. $<-1.50$ & & 140 & 97 & 22 \\
\hline and F.C. $>1.66$ & \multirow{2}{*}{351} & 119 & 161 & 94 \\
\hline and F.C. $<-1.66$ & & 71 & 45 & 7 \\
\hline and F.C. $>2.00$ & \multirow{2}{*}{173} & 70 & 95 & 39 \\
\hline and F.C. $<-2.00$ & & 24 & 14 & 3 \\
\hline and F.C. $>2.50$ & \multirow{2}{*}{79} & 36 & 38 & 19 \\
\hline and F.C. $<-2.50$ & & 6 & 8 & 1 \\
\hline
\end{tabular}

Significance was determined by $t$-test and Z-score as detailed in Materials and Methods from three biological replicates. The 173 specific proteins whose levels were altered $>2.0$-fold in either direction are listed in Table 2. 


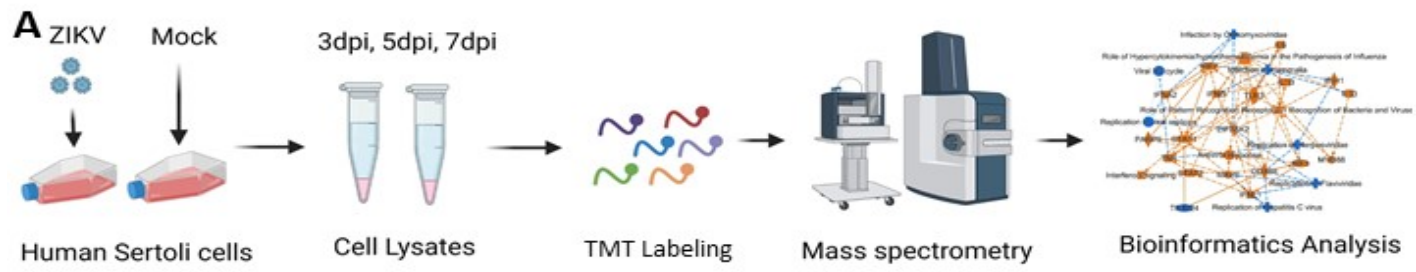

B

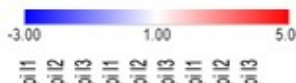

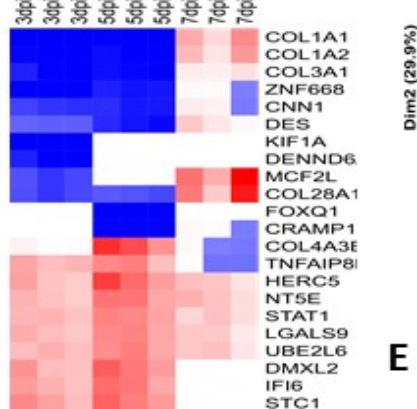

$\mathbf{E}$

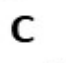

C
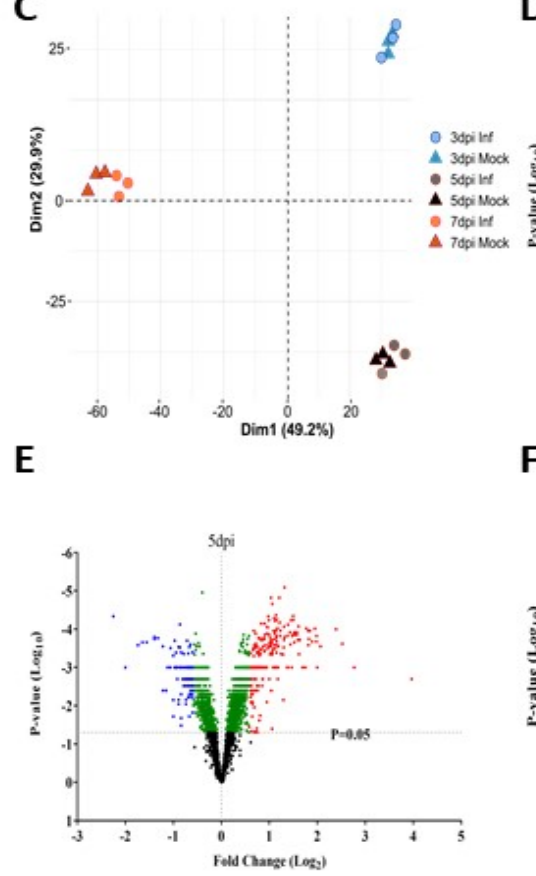

G

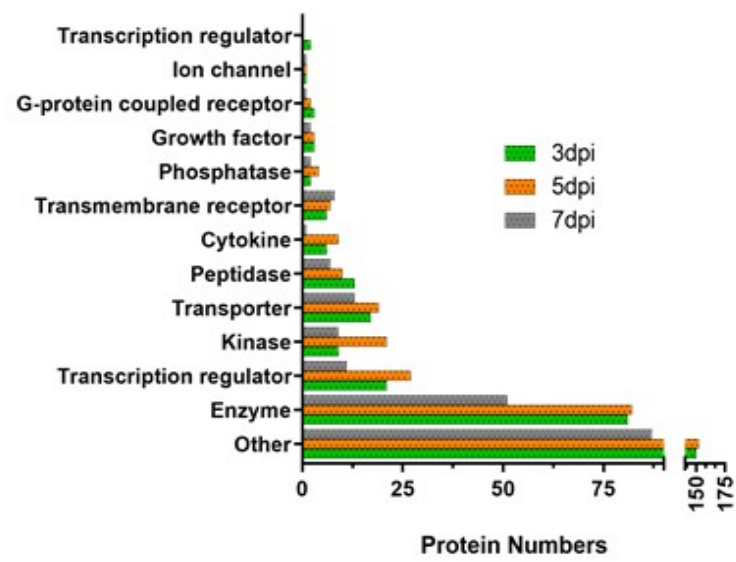

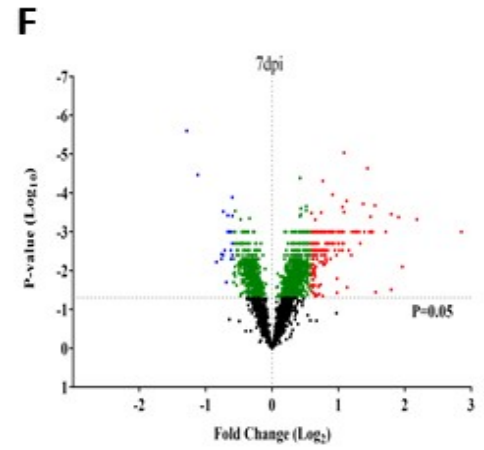

D

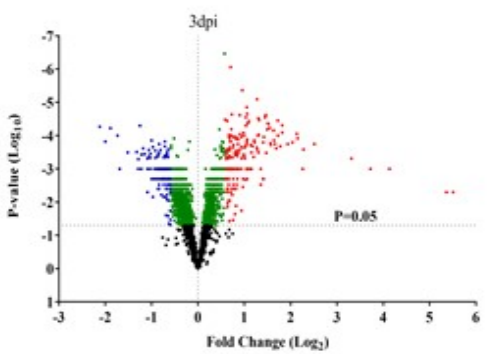

$\mathbf{F}$

Figure 2. Detection of cellular protein level alterations in ZIKV-infected Sertoli cells by mass spectrometry. (A) Schematic flow diagram of the study design. (B) Heatmap of most affected proteins (Fold change $\geq 2.5$ or $\leq-2.5$; $p$ value $<0.05$ ) by ZIKV infection in Sertoli cells. Red and blue colors indicate up-regulation and down-regulation, respectively. (C) PCA plot of proteomic data from mock- and ZIKV-infected cells, from all three replicates. Volcano plots displaying the protein level alterations after ZIKV infection at $3 \mathrm{dpi}$ (D), $5 \mathrm{dpi}$ (E), and $7 \mathrm{dpi}(\mathbf{F})$. Red = significantly up-regulated $($ FC $>1.5 ; p$-value $<0.05)$, blue = significantly down-regulated $(\mathrm{FC}<-1.5 ; p$-value $<0.05)$, green = significantly affected ( $p$-value $<0.05$, but FC $< \pm 1.5$ ), black = not significantly affected ( $p$-value $>0.05)$. (G) Classification of significantly altered (Fold change $\geq 1.5$ or $\leq-1.5$; $p$ value $<0.05$ ) protein types at different time points after ZIKV infection. Abbreviations. FC = Fold change; dpi = Days post infection; inf = Infected. 
Table 2. List of protein levels altered at least 2 -fold and significant by $t$-test ( $p$-value $<0.05)$ or Z-score $(\geq 1.96 \sigma$ or $\leq-1.96 \sigma)$.

\begin{tabular}{|c|c|c|c|c|c|c|c|c|}
\hline \multirow{2}{*}{ Swprot } & \multirow{2}{*}{ Gene } & \multirow{2}{*}{ Protein } & \multicolumn{2}{|c|}{3 dpi } & \multicolumn{2}{|c|}{$5 \mathrm{dpi}$} & \multicolumn{2}{|c|}{$7 \mathrm{dpi}$} \\
\hline & & & Inf/Mock F.C. & $p$-Value & Inf/Mock F.C. & $p$-Value & Inf/Mock F.C. & $p$-Value \\
\hline \multicolumn{9}{|c|}{ Up-regulated proteins } \\
\hline O15539 & RGS5 & Regulator of G-protein signaling 5 & 45.46 & $5.5 \times 10^{-3}$ & & & & \\
\hline Q86XK2 & FBXO11 & F-box only protein 11 & 41.16 & $4.9 \times 10^{-3}$ & -1.01 & $7.9 \times 10^{-1}$ & 1.11 & $1.9 \times 10^{-1}$ \\
\hline Q96PU4 & UHRF2 & E3 ubiquitin-protein ligase UHRF2 & 17.55 & $1.1 \times 10^{-3}$ & 5.74 & $2.4 \times 10^{-4}$ & -1.10 & $2.5 \times 10^{-1}$ \\
\hline Q12955 & ANK3 & Ankyrin-3 & 13.21 & $8.1 \times 10^{-4}$ & 3.36 & $6.5 \times 10^{-4}$ & 2.29 & $3.8 \times 10^{-3}$ \\
\hline Q8N6Y2 & LRRC17 & Leucine-rich repeat-containing protein 17 & 9.94 & $4.9 \times 10^{-4}$ & 2.82 & $1.4 \times 10^{-4}$ & 1.31 & $9.5 \times 10^{-3}$ \\
\hline O15544 & LINC01565 & Protein GR6 & 5.71 & $1.8 \times 10^{-4}$ & & & & \\
\hline P14679 & TYR & Tyrosinase & 4.85 & $2.6 \times 10^{-4}$ & 15.63 & $1.8 \times 10^{-3}$ & & \\
\hline O95294 & RASAL1 & RasGAP-activating-like protein 1 & 4.80 & $8.5 \times 10^{-4}$ & 2.08 & $4.0 \times 10^{-2}$ & & \\
\hline Q9P275 & USP36 & Ubiquitin carboxyl-terminal hydrolase 36 & 4.44 & $1.2 \times 10^{-4}$ & 1.03 & $7.0 \times 10^{-1}$ & 1.06 & $3.5 \times 10^{-1}$ \\
\hline Q96PN6 & ADCY10 & Adenylate cyclase type 10 & 4.39 & $8.8 \times 10^{-5}$ & 4.19 & $2.8 \times 10^{-4}$ & 1.00 & $9.1 \times 10^{-1}$ \\
\hline P61371 & ISL1 & Insulin gene enhancer protein ISL-1 & 4.11 & $1.6 \times 10^{-4}$ & & & & \\
\hline P10145 & CXCL8 & Interleukin-8 & 3.83 & $1.8 \times 10^{-4}$ & 3.07 & $4.5 \times 10^{-4}$ & & \\
\hline P20591 & MX1 & Interferon-induced GTP-binding protein $\mathrm{Mx1}$ & 3.53 & $8.1 \times 10^{-5}$ & 5.24 & $1.0 \times 10^{-4}$ & 4.54 & $4.8 \times 10^{-4}$ \\
\hline Q6ZV65 & FAM47E & Protein FAM47E & 3.53 & $4.9 \times 10^{-5}$ & & & & \\
\hline Q13438 & OS9 & Protein & 3.42 & $2.1 \times 10^{-4}$ & 0.99 & $8.0 \times 10^{-1}$ & 1.04 & $5.7 \times 10^{-1}$ \\
\hline Q8N9S9 & SNX31 & Sorting nexin-31 & 3.31 & $1.0 \times 10^{-4}$ & & & & \\
\hline O14879 & IFIT3 & $\begin{array}{l}\text { Interferon-induced protein with } \\
\text { tetratricopeptide repeats } 3\end{array}$ & 3.30 & $3.5 \times 10^{-5}$ & 3.41 & $9.5 \times 10^{-5}$ & 3.75 & $4.2 \times 10^{-4}$ \\
\hline Q8TEJ3 & SH3RF3 & SH3 domain-containing RING finger protein 3 & 3.22 & $2.4 \times 10^{-4}$ & 1.01 & $8.1 \times 10^{-1}$ & & \\
\hline P30447 & HLA-A & $\begin{array}{l}\text { HLA class I histocompatibility antigen, A-23 } \\
\text { alpha chain }\end{array}$ & 3.17 & $1.8 \times 10^{-4}$ & 3.96 & $1.5 \times 10^{-4}$ & & \\
\hline
\end{tabular}


Table 2. Cont.

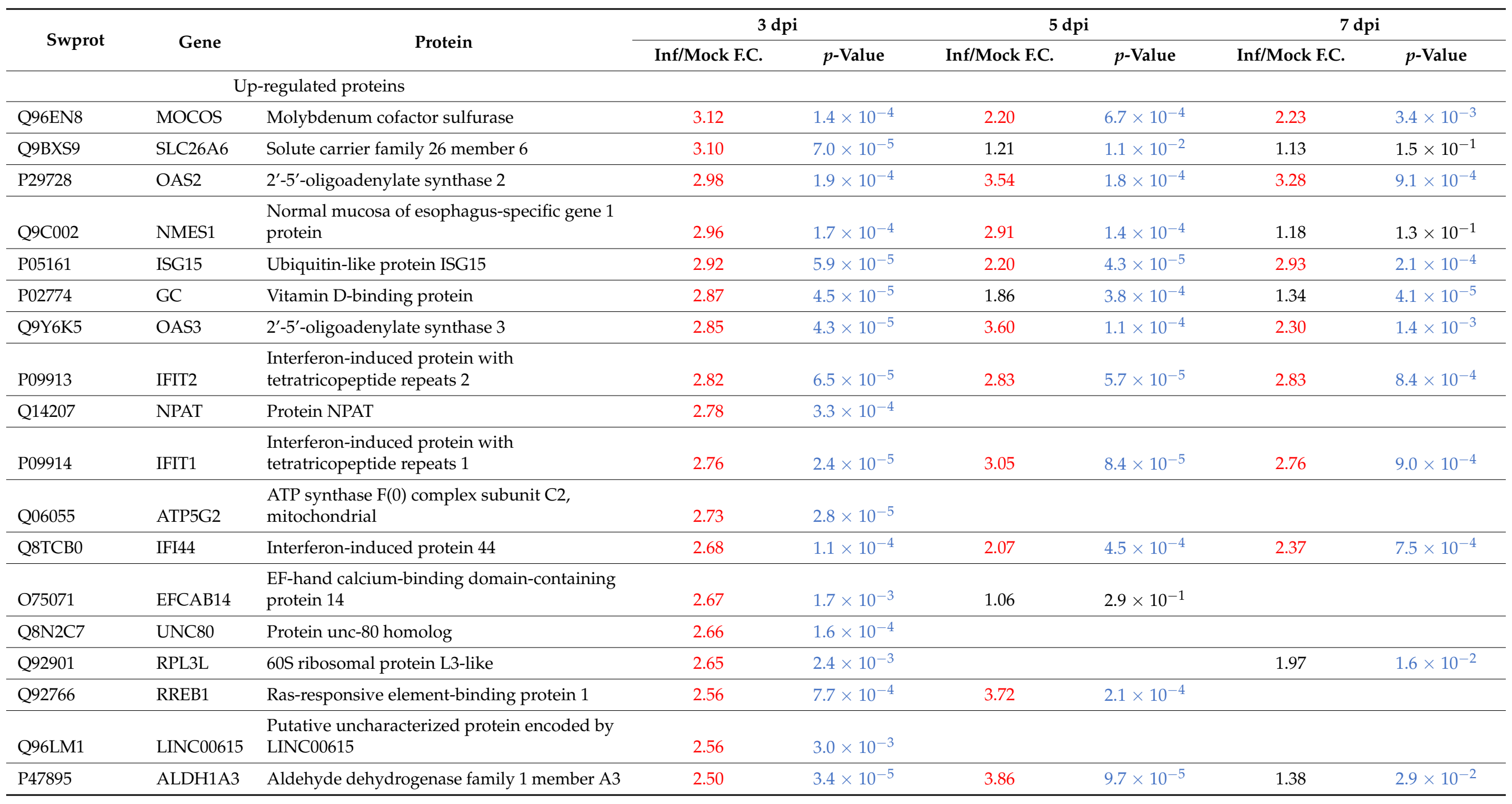


Table 2. Cont.

\begin{tabular}{|c|c|c|c|c|c|c|c|c|}
\hline \multirow{2}{*}{ Swprot } & \multirow{2}{*}{ Gene } & \multirow{2}{*}{ Protein } & \multicolumn{2}{|c|}{3 dpi } & \multicolumn{2}{|c|}{5 dpi } & \multicolumn{2}{|c|}{$7 \mathrm{dpi}$} \\
\hline & & & Inf/Mock F.C. & $p$-Value & Inf/Mock F.C. & $p$-Value & Inf/Mock F.C. & $p$-Value \\
\hline \multicolumn{9}{|c|}{ Up-regulated proteins } \\
\hline P20592 & MX2 & Interferon-induced GTP-binding protein $\mathrm{Mx} 2$ & 2.48 & $9.3 \times 10^{-5}$ & 2.98 & $1.2 \times 10^{-4}$ & 2.80 & $4.1 \times 10^{-4}$ \\
\hline Q96AZ6 & ISG20 & Interferon-stimulated gene $20 \mathrm{kDa}$ protein & 2.47 & $3.0 \times 10^{-5}$ & 2.46 & $3.0 \times 10^{-4}$ & & \\
\hline Q9BXU1 & STK31 & Serine/threonine-protein kinase 31 & 2.47 & $3.2 \times 10^{-4}$ & & & & \\
\hline Q9Y3Z3 & SAMHD1 & $\begin{array}{l}\text { Deoxynucleoside triphosphate } \\
\text { triphosphohydrolase SAMHD1 }\end{array}$ & 2.46 & $1.0 \times 10^{-4}$ & 2.82 & $4.5 \times 10^{-5}$ & 2.49 & $1.1 \times 10^{-3}$ \\
\hline P52823 & STC1 & Stanniocalcin-1 & 2.43 & $2.6 \times 10^{-4}$ & 2.98 & $1.4 \times 10^{-4}$ & & \\
\hline P02795 & MT2A & Metallothionein-2 & 2.42 & $8.0 \times 10^{-6}$ & 6.81 & $6.8 \times 10^{-4}$ & & \\
\hline P30464 & HLA-B & $\begin{array}{l}\text { HLA class I histocompatibility antigen, B-15 } \\
\text { alpha chain }\end{array}$ & 2.41 & $2.3 \times 10^{-4}$ & 2.49 & $1.6 \times 10^{-4}$ & 2.11 & $1.5 \times 10^{-3}$ \\
\hline A6ND36 & FAM83G & Protein FAM83G & 2.33 & $1.6 \times 10^{-4}$ & 1.77 & $1.9 \times 10^{-4}$ & 1.14 & $7.0 \times 10^{-4}$ \\
\hline P48307 & TFPI2 & Tissue factor pathway inhibitor 2 & 2.32 & $1.4 \times 10^{-4}$ & 3.11 & $1.9 \times 10^{-4}$ & 1.18 & $4.1 \times 10^{-2}$ \\
\hline Q6UXH9 & PAMR1 & Inactive serine protease PAMR1 & 2.26 & $2.1 \times 10^{-4}$ & 1.12 & $7.5 \times 10^{-2}$ & & \\
\hline P09912 & IFI6 & Interferon alpha-inducible protein 6 & 2.23 & $5.0 \times 10^{-4}$ & 2.87 & $2.2 \times 10^{-4}$ & & \\
\hline Q71F56 & MED13L & $\begin{array}{l}\text { Mediator of RNA polymerase II transcription } \\
\text { subunit 13-like }\end{array}$ & 2.23 & $3.2 \times 10^{-4}$ & & & 1.04 & $8.1 \times 10^{-1}$ \\
\hline Q8TDJ6 & DMXL2 & DmX-like protein 2 & 2.21 & $1.1 \times 10^{-3}$ & 3.02 & $1.9 \times 10^{-4}$ & & \\
\hline Q6P589 & TNFAIP8L2 & $\begin{array}{l}\text { Tumor necrosis factor alpha-induced protein } \\
\text { 8-like protein } 2\end{array}$ & 2.20 & $1.9 \times 10^{-4}$ & 2.59 & $6.9 \times 10^{-4}$ & -1.13 & $2.2 \times 10^{-1}$ \\
\hline P18464 & HLA-B & $\begin{array}{l}\text { HLA class I histocompatibility antigen, B-51 } \\
\text { alpha chain }\end{array}$ & 2.18 & $7.6 \times 10^{-4}$ & 2.78 & $1.5 \times 10^{-4}$ & 2.62 & $9.1 \times 10^{-4}$ \\
\hline Q10589 & BST2 & Bone marrow stromal antigen 2 & 2.14 & $3.7 \times 10^{-5}$ & 3.95 & $2.1 \times 10^{-4}$ & 3.47 & $3.5 \times 10^{-4}$ \\
\hline P01033 & TIMP1 & Metalloproteinase inhibitor 1 & 2.11 & $4.2 \times 10^{-5}$ & 2.02 & $4.5 \times 10^{-4}$ & 1.11 & $1.3 \times 10^{-1}$ \\
\hline
\end{tabular}


Table 2. Cont.

\begin{tabular}{|c|c|c|c|c|c|c|c|c|}
\hline \multirow{2}{*}{ Swprot } & \multirow{2}{*}{ Gene } & \multirow{2}{*}{ Protein } & \multicolumn{2}{|c|}{3 dpi } & \multicolumn{2}{|c|}{5 dpi } & \multicolumn{2}{|c|}{$7 \mathrm{dpi}$} \\
\hline & & & Inf/Mock F.C. & $p$-Value & Inf/Mock F.C. & $p$-Value & Inf/Mock F.C. & $p$-Value \\
\hline \multicolumn{9}{|c|}{ Up-regulated proteins } \\
\hline Q96J88 & EPSTI1 & Epithelial-stromal interaction protein 1 & 2.10 & $8.8 \times 10^{-4}$ & & & 1.57 & $7.8 \times 10^{-3}$ \\
\hline O14933 & UBE2L6 & Ubiquitin/ISG15-conjugating enzyme E2 L6 & 2.09 & $2.2 \times 10^{-5}$ & 2.71 & $2.5 \times 10^{-4}$ & 1.73 & $7.4 \times 10^{-3}$ \\
\hline P30685 & HLA-B & $\begin{array}{l}\text { HLA class I histocompatibility antigen, B- } 35 \\
\text { alpha chain }\end{array}$ & 2.09 & $1.3 \times 10^{-4}$ & 2.28 & $2.8 \times 10^{-4}$ & 2.00 & $2.0 \times 10^{-3}$ \\
\hline P42224 & STAT1 & $\begin{array}{l}\text { Signal transducer and activator of } \\
\text { transcription 1-alpha/beta }\end{array}$ & 2.08 & $1.4 \times 10^{-5}$ & 2.74 & $5.8 \times 10^{-5}$ & 1.71 & $3.3 \times 10^{-3}$ \\
\hline P15407 & FOSL1 & Fos-related antigen 1 & 2.08 & $1.5 \times 10^{-4}$ & 2.18 & $3.2 \times 10^{-4}$ & & \\
\hline O14791 & APOL1 & Apolipoprotein L1 & 2.07 & $5.5 \times 10^{-4}$ & & & & \\
\hline Q9UII4 & HERC5 & E3 ISG15-protein ligase HERC5 & 2.07 & $5.5 \times 10^{-4}$ & 3.23 & $5.5 \times 10^{-4}$ & 1.75 & $3.4 \times 10^{-3}$ \\
\hline O00182 & LGALS9 & Galectin-9 & 2.07 & $1.8 \times 10^{-4}$ & 2.69 & $1.2 \times 10^{-4}$ & 1.67 & $3.9 \times 10^{-3}$ \\
\hline Q8IXQ6 & PARP9 & Poly [ADP-ribose] polymerase 9 & 2.05 & $2.8 \times 10^{-5}$ & 2.11 & $3.3 \times 10^{-4}$ & 1.77 & $1.6 \times 10^{-3}$ \\
\hline Q03405 & PLAUR & $\begin{array}{l}\text { Urokinase plasminogen activator surface } \\
\text { receptor }\end{array}$ & 2.05 & $5.0 \times 10^{-5}$ & 1.53 & $2.0 \times 10^{-3}$ & 1.41 & $7.8 \times 10^{-4}$ \\
\hline Q96L93 & KIF16B & Kinesin-like protein KIF16B & 2.04 & $2.1 \times 10^{-3}$ & & & 1.13 & $1.4 \times 10^{-1}$ \\
\hline Q29960 & HLA-C & $\begin{array}{l}\text { HLA class I histocompatibility antigen, Cw-16 } \\
\text { alpha chain }\end{array}$ & 2.04 & $2.1 \times 10^{-4}$ & 2.37 & $4.4 \times 10^{-4}$ & & \\
\hline P25774 & CTSS & Cathepsin S & 2.02 & $4.8 \times 10^{-4}$ & 1.79 & $3.3 \times 10^{-4}$ & & \\
\hline P04733 & MT1F & Metallothionein-1F & & & 3.99 & $5.9 \times 10^{-4}$ & & \\
\hline Q9Y5P4 & COL4A3BP & Collagen type IV alpha-3-binding protein & 1.08 & $1.3 \times 10^{-1}$ & 3.52 & $2.6 \times 10^{-4}$ & -1.03 & $5.5 \times 10^{-1}$ \\
\hline P05231 & IL6 & Interleukin-6 & & & 3.51 & $1.1 \times 10^{-4}$ & & \\
\hline P21589 & NT5E & 5'-nucleotidase & 1.98 & $8.2 \times 10^{-5}$ & 2.87 & $1.2 \times 10^{-4}$ & 1.88 & $1.6 \times 10^{-3}$ \\
\hline
\end{tabular}


Table 2. Cont.

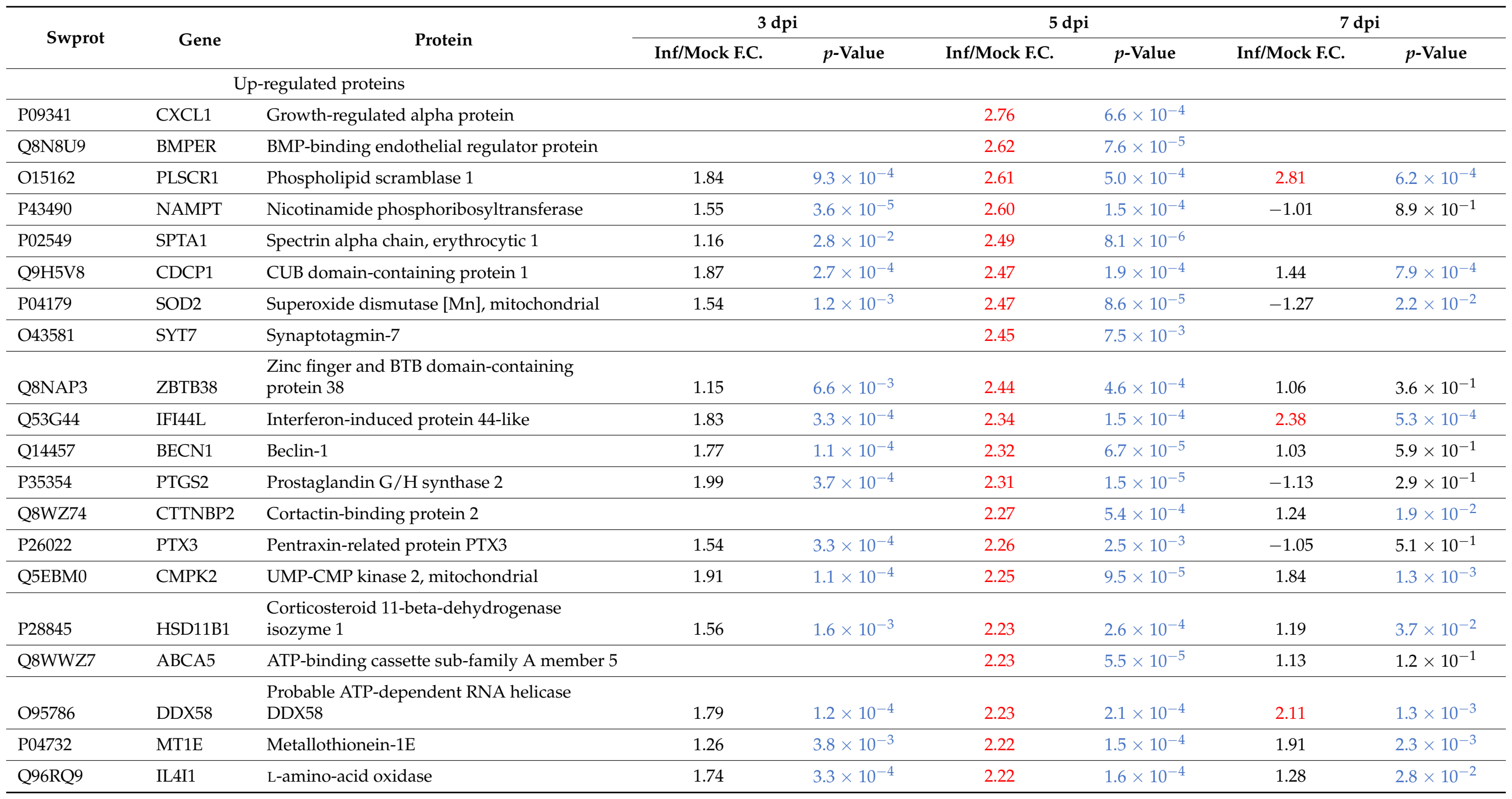


Table 2. Cont.

\begin{tabular}{|c|c|c|c|c|c|c|c|c|}
\hline \multirow{2}{*}{ Swprot } & \multirow{2}{*}{ Gene } & \multirow{2}{*}{ Protein } & \multicolumn{2}{|c|}{$3 \mathrm{dpi}$} & \multicolumn{2}{|c|}{$5 \mathrm{dpi}$} & \multicolumn{2}{|c|}{$7 \mathrm{dpi}$} \\
\hline & & & Inf/Mock F.C. & $p$-Value & Inf/Mock F.C. & $p$-Value & Inf/Mock F.C. & $p$-Value \\
\hline \multicolumn{9}{|c|}{ Up-regulated proteins } \\
\hline P01892 & HLA-A & $\begin{array}{l}\text { HLA class I histocompatibility antigen, A-2 } \\
\text { alpha chain }\end{array}$ & 1.78 & $2.8 \times 10^{-4}$ & 2.22 & $4.1 \times 10^{-4}$ & 1.60 & $4.9 \times 10^{-3}$ \\
\hline Q4VCS5 & AMOT & Angiomotin & 1.92 & $4.8 \times 10^{-4}$ & 2.20 & $1.1 \times 10^{-4}$ & & \\
\hline P00973 & OAS1 & $2 '-5$-oligoadenylate synthase 1 & 1.99 & $1.0 \times 10^{-4}$ & 2.20 & $1.3 \times 10^{-3}$ & 2.02 & $3.5 \times 10^{-3}$ \\
\hline Q96C10 & DHX58 & $\begin{array}{l}\text { Probable ATP-dependent RNA helicase } \\
\text { DHX58 }\end{array}$ & 1.35 & $1.6 \times 10^{-2}$ & 2.18 & $1.8 \times 10^{-4}$ & 1.08 & $2.6 \times 10^{-1}$ \\
\hline P13500 & CCL2 & C-C motif chemokine 2 & & & 2.17 & $7.9 \times 10^{-4}$ & & \\
\hline Q9BY76 & ANGPTL4 & Angiopoietin-related protein 4 & 1.58 & $4.7 \times 10^{-4}$ & 2.17 & $8.3 \times 10^{-4}$ & 1.36 & $1.0 \times 10^{-2}$ \\
\hline Q9Y6I4 & USP3 & Ubiquitin carboxyl-terminal hydrolase 3 & 1.01 & $7.1 \times 10^{-1}$ & 2.16 & $1.3 \times 10^{-4}$ & & \\
\hline O94808 & GFPT2 & $\begin{array}{l}\text { Glutamine-fructose-6-phosphate } \\
\text { aminotransferase [isomerizing] } 2\end{array}$ & 1.65 & $6.4 \times 10^{-5}$ & 2.15 & $2.6 \times 10^{-4}$ & 1.38 & $6.8 \times 10^{-3}$ \\
\hline P15153 & RAC2 & Ras-related C 3 botulinum toxin substrate 2 & 1.58 & $2.7 \times 10^{-4}$ & 2.14 & $5.2 \times 10^{-5}$ & 1.04 & $3.8 \times 10^{-1}$ \\
\hline P19525 & EIF2AK2 & $\begin{array}{l}\text { Interferon-induced, double-stranded } \\
\text { RNA-activated protein kinase }\end{array}$ & 1.59 & $1.1 \times 10^{-4}$ & 2.13 & $1.1 \times 10^{-4}$ & 1.91 & $1.6 \times 10^{-3}$ \\
\hline Q9HB58 & SP110 & Sp110 nuclear body protein & 1.80 & $1.7 \times 10^{-3}$ & 2.11 & $9.3 \times 10^{-4}$ & 1.81 & $1.2 \times 10^{-3}$ \\
\hline P05534 & HLA-A & $\begin{array}{l}\text { HLA class I histocompatibility antigen, A- } 24 \\
\text { alpha chain }\end{array}$ & 1.58 & $4.0 \times 10^{-4}$ & 2.09 & $1.9 \times 10^{-4}$ & 1.98 & $8.2 \times 10^{-4}$ \\
\hline P52926 & HMGA2 & High mobility group protein HMGI-C & 1.28 & $2.5 \times 10^{-2}$ & 2.09 & $2.2 \times 10^{-5}$ & -1.16 & $5.7 \times 10^{-2}$ \\
\hline O75508 & CLDN11 & Claudin-11 & 1.05 & $5.9 \times 10^{-1}$ & 2.09 & $3.9 \times 10^{-4}$ & 1.27 & $2.1 \times 10^{-3}$ \\
\hline P07148 & FABP1 & Fatty acid-binding protein, liver & & & 2.09 & $4.4 \times 10^{-4}$ & -1.09 & $4.6 \times 10^{-1}$ \\
\hline Q7Z402 & TMC7 & Transmembrane channel-like protein 7 & & & 2.08 & $2.3 \times 10^{-4}$ & & \\
\hline
\end{tabular}


Table 2. Cont.

\begin{tabular}{|c|c|c|c|c|c|c|c|c|}
\hline \multirow{2}{*}{ Swprot } & \multirow{2}{*}{ Gene } & \multirow{2}{*}{ Protein } & \multicolumn{2}{|c|}{$3 \mathrm{dpi}$} & \multicolumn{2}{|c|}{$5 \mathrm{dpi}$} & \multicolumn{2}{|c|}{$7 \mathrm{dpi}$} \\
\hline & & & Inf/Mock F.C. & $p$-Value & Inf/Mock F.C. & $p$-Value & Inf/Mock F.C. & $p$-Value \\
\hline \multicolumn{9}{|c|}{ Up-regulated proteins } \\
\hline Q01973 & ROR1 & $\begin{array}{l}\text { Inactive tyrosine-protein kinase } \\
\text { transmembrane receptor ROR1 }\end{array}$ & & & 2.08 & $4.6 \times 10^{-4}$ & 1.41 & $2.1 \times 10^{-3}$ \\
\hline Q8IVT2 & MISP & Mitotic interactor and substrate of PLK1 & 1.75 & $3.8 \times 10^{-4}$ & 2.07 & $4.0 \times 10^{-4}$ & & \\
\hline Q3MIT2 & PUS10 & Putative tRNA pseudouridine synthase Pus10 & & & 2.06 & $4.3 \times 10^{-3}$ & -1.31 & $5.5 \times 10^{-3}$ \\
\hline Q15646 & OASL & 2'-5'-oligoadenylate synthase-like protein & 1.82 & $1.3 \times 10^{-4}$ & 2.05 & $2.8 \times 10^{-4}$ & 1.99 & $1.8 \times 10^{-3}$ \\
\hline P01584 & IL1B & Interleukin-1 beta & 1.88 & $9.0 \times 10^{-5}$ & 2.04 & $2.1 \times 10^{-4}$ & & \\
\hline Q7Z3S9 & NOTCH2NL & Notch homolog 2 N-terminal-like protein & 1.13 & $1.0 \times 10^{-1}$ & 2.02 & $1.7 \times 10^{-4}$ & 1.03 & $6.1 \times 10^{-1}$ \\
\hline P68871 & HBB & Hemoglobin subunit beta & 1.54 & $3.6 \times 10^{-3}$ & 2.02 & $2.4 \times 10^{-4}$ & 1.31 & $1.3 \times 10^{-2}$ \\
\hline Q9BYX4 & IFIH1 & $\begin{array}{l}\text { Interferon-induced helicase C } \\
\text { domain-containing protein } 1\end{array}$ & 1.82 & $1.8 \times 10^{-4}$ & 2.01 & $2.5 \times 10^{-4}$ & 2.09 & $2.7 \times 10^{-3}$ \\
\hline P28838 & LAP3 & Cytosol aminopeptidase & 1.66 & $2.3 \times 10^{-5}$ & 2.00 & $1.4 \times 10^{-4}$ & 1.42 & $6.2 \times 10^{-3}$ \\
\hline Q92597 & NDRG1 & Protein NDRG1 & 1.21 & $1.6 \times 10^{-3}$ & 2.00 & $1.4 \times 10^{-4}$ & 1.75 & $7.5 \times 10^{-4}$ \\
\hline P11166 & SLC2A1 & $\begin{array}{l}\text { Solute carrier family 2, facilitated glucose } \\
\text { transporter member } 1\end{array}$ & 1.65 & $6.2 \times 10^{-4}$ & 1.91 & $7.9 \times 10^{-4}$ & 2.12 & $9.2 \times 10^{-6}$ \\
\hline P09871 & C1S & Complement $\mathrm{C} 1$ s subcomponent & 1.08 & $2.3 \times 10^{-1}$ & 1.25 & $5.7 \times 10^{-3}$ & 2.10 & $1.3 \times 10^{-3}$ \\
\hline P11169 & SLC2A3 & $\begin{array}{l}\text { Solute carrier family 2, facilitated glucose } \\
\text { transporter member } 3\end{array}$ & 1.28 & $3.2 \times 10^{-3}$ & 1.21 & $2.2 \times 10^{-2}$ & 2.71 & $2.3 \times 10^{-5}$ \\
\hline Q96DE5 & ANAPC16 & Anaphase-promoting complex subunit 16 & -1.12 & $2.0 \times 10^{-1}$ & 1.13 & $8.3 \times 10^{-3}$ & 3.57 & $4.4 \mathrm{E}-10$ \\
\hline P00966 & ASS1 & Argininosuccinate synthase & -1.12 & $1.1 \times 10^{-1}$ & -1.13 & $2.1 \times 10^{-2}$ & 2.08 & $1.6 \times 10^{-3}$ \\
\hline Q2UY09 & COL28A1 & Collagen alpha- $1(X X V I I I)$ chain & -1.93 & $1.2 \times 10^{-3}$ & -1.71 & $3.1 \times 10^{-3}$ & 2.95 & $3.6 \times 10^{-2}$ \\
\hline
\end{tabular}


Table 2. Cont.

\begin{tabular}{|c|c|c|c|c|c|c|c|c|}
\hline \multirow{2}{*}{ Swprot } & \multirow{2}{*}{ Gene } & \multirow{2}{*}{ Protein } & \multicolumn{2}{|c|}{$3 \mathrm{dpi}$} & \multicolumn{2}{|c|}{$5 \mathrm{dpi}$} & \multicolumn{2}{|c|}{7 dpi } \\
\hline & & & Inf/Mock F.C. & $p$-Value & Inf/Mock F.C. & $p$-Value & Inf/Mock F.C. & $p$-Value \\
\hline \multicolumn{9}{|c|}{ Up-regulated proteins } \\
\hline P04264 & KRT1 & Keratin, type II cytoskeletal 1 & -1.40 & $1.9 \times 10^{-1}$ & -1.98 & $1.9 \times 10^{-2}$ & -2.41 & $2.5 \times 10^{-6}$ \\
\hline Q9HCJ2 & LRRC4C & Leucine-rich repeat-containing protein $4 \mathrm{C}$ & & & & & 7.23 & $1.3 \times 10^{-3}$ \\
\hline Q8IXR9 & C12orf56 & Uncharacterized protein C12orf56 & & & & & 3.90 & $8.3 \times 10^{-3}$ \\
\hline O15068 & MCF2L & Guanine nucleotide exchange factor DBS & -1.93 & $1.6 \times 10^{-3}$ & & & 3.47 & $3.1 \times 10^{-2}$ \\
\hline Q9NQ90 & ANO2 & Anoctamin-2 & 1.23 & $2.1 \times 10^{-2}$ & & & 2.86 & $1.2 \times 10^{-3}$ \\
\hline P17693 & HLA-G & $\begin{array}{l}\text { HLA class I histocompatibility antigen, alpha } \\
\text { chain G }\end{array}$ & & & & & 2.59 & $1.9 \times 10^{-4}$ \\
\hline Q8IZ26 & ZNF34 & Zinc finger protein 34 & & & & & 2.51 & $2.2 \times 10^{-3}$ \\
\hline Q9Y225 & RNF24 & RING finger protein 24 & & & 1.13 & $5.7 \times 10^{-2}$ & 2.42 & $8.5 \times 10^{-4}$ \\
\hline P04222 & HLA-C & $\begin{array}{l}\text { HLA class I histocompatibility antigen, } \mathrm{Cw}-3 \\
\text { alpha chain }\end{array}$ & & & & & 2.18 & $1.6 \times 10^{-4}$ \\
\hline Q96B67 & ARRDC3 & Arrestin domain-containing protein 3 & & & & & 2.09 & $2.3 \times 10^{-4}$ \\
\hline Q13772 & NCOA4 & Nuclear receptor coactivator 4 & & & -1.04 & $6.0 \times 10^{-1}$ & 2.08 & $1.3 \times 10^{-3}$ \\
\hline Q13137 & CALCOCO2 & $\begin{array}{l}\text { Calcium-binding and coiled-coil } \\
\text { domain-containing protein } 2\end{array}$ & 1.19 & $3.4 \times 10^{-2}$ & -1.04 & $4.1 \times 10^{-1}$ & 2.03 & $7.2 \times 10^{-4}$ \\
\hline \multicolumn{9}{|c|}{ Down-regulated proteins } \\
\hline Q12756 & KIF1A & Kinesin-like protein KIF1A & -4.38 & $5.4 \times 10^{-5}$ & & & & \\
\hline P02452 & COL1A1 & Collagen alpha-1(I) chain & -3.95 & $1.5 \times 10^{-4}$ & -3.39 & $2.6 \times 10^{-4}$ & 2.19 & $2.7 \times 10^{-2}$ \\
\hline Q96K58 & ZNF668 & Zinc finger protein 668 & -3.66 & $6.0 \times 10^{-5}$ & -2.63 & $1.6 \times 10^{-4}$ & 1.03 & $3.6 \times 10^{-1}$ \\
\hline P08123 & COL1A2 & Collagen alpha-2(I) chain & -3.39 & $1.0 \times 10^{-4}$ & -3.15 & $2.2 \times 10^{-4}$ & 1.97 & $3.7 \times 10^{-2}$ \\
\hline Q8IWF6 & DENND6A & Protein DENND6A & -3.20 & $6.3 \times 10^{-4}$ & & & & \\
\hline
\end{tabular}


Table 2. Cont.

\begin{tabular}{|c|c|c|c|c|c|c|c|c|}
\hline \multirow{2}{*}{ Swprot } & \multirow{2}{*}{ Gene } & \multirow{2}{*}{ Protein } & \multicolumn{2}{|c|}{3 dpi } & \multicolumn{2}{|c|}{$5 \mathrm{dpi}$} & \multicolumn{2}{|c|}{$7 \mathrm{dpi}$} \\
\hline & & & Inf/Mock F.C. & $p$-Value & Inf/Mock F.C. & $p$-Value & Inf/Mock F.C. & $p$-Value \\
\hline \multicolumn{9}{|c|}{ Down-regulated proteins } \\
\hline P02461 & COL3A1 & Collagen alpha-1(III) chain & -2.87 & $3.2 \times 10^{-4}$ & -2.97 & $2.2 \times 10^{-4}$ & 1.33 & $1.1 \times 10^{-2}$ \\
\hline Q9H1P3 & OSBPL2 & Oxysterol-binding protein-related protein 2 & -2.47 & $3.1 \times 10^{-4}$ & 1.41 & $4.9 \times 10^{-3}$ & & \\
\hline Q8IZX4 & TAF1L & $\begin{array}{l}\text { Transcription initiation factor TFIID subunit } \\
\text { 1-like }\end{array}$ & -2.46 & $5.1 \times 10^{-4}$ & & & -1.69 & $3.8 \times 10^{-3}$ \\
\hline Q96JG9 & ZNF469 & Zinc finger protein 469 & -2.41 & $5.0 \times 10^{-5}$ & & & 1.03 & $7.2 \times 10^{-1}$ \\
\hline P52732 & KIF11 & Kinesin-like protein KIF11 & -2.34 & $6.7 \times 10^{-4}$ & & & & \\
\hline P09486 & SPARC & SPARC & -2.32 & $3.7 \times 10^{-4}$ & -2.08 & $9.4 \times 10^{-4}$ & 1.41 & $9.8 \times 10^{-3}$ \\
\hline P20908 & COL5A1 & Collagen alpha- $1(\mathrm{~V})$ chain & -2.29 & $2.4 \times 10^{-4}$ & -1.99 & $4.9 \times 10^{-4}$ & 1.40 & $4.5 \times 10^{-2}$ \\
\hline Q96PQ7 & KLHL5 & Kelch-like protein 5 & -2.20 & $3.6 \times 10^{-4}$ & -1.11 & $1.2 \times 10^{-1}$ & & \\
\hline Q8N7X1 & RBMXL3 & RNA-binding motif protein, $\mathrm{X}$-linked-like-3 & -2.13 & $5.0 \times 10^{-3}$ & -2.22 & $4.0 \times 10^{-3}$ & & \\
\hline O00767 & SCD & Acyl-CoA desaturase & -2.13 & $4.9 \times 10^{-4}$ & -1.69 & $2.1 \times 10^{-3}$ & 1.31 & $5.7 \times 10^{-3}$ \\
\hline P61916 & NPC2 & Epididymal secretory protein E1 & -2.10 & $4.3 \times 10^{-4}$ & -2.09 & $3.1 \times 10^{-4}$ & -1.52 & $4.1 \times 10^{-3}$ \\
\hline Q05682 & CALD1 & Caldesmon & -2.08 & $2.7 \times 10^{-4}$ & -1.95 & $6.8 \times 10^{-4}$ & -1.15 & $1.1 \times 10^{-1}$ \\
\hline Q8N806 & UBR7 & Putative E3 ubiquitin-protein ligase UBR7 & -2.06 & $1.3 \times 10^{-3}$ & 1.47 & $2.6 \times 10^{-3}$ & 1.03 & $6.3 \times 10^{-1}$ \\
\hline Q15113 & PCOLCE & Procollagen C-endopeptidase enhancer 1 & -2.06 & $4.6 \times 10^{-4}$ & -1.65 & $7.8 \times 10^{-4}$ & 1.39 & $1.7 \times 10^{-2}$ \\
\hline O75094 & SLIT3 & Slit homolog 3 protein & -2.04 & $3.5 \times 10^{-4}$ & & & & \\
\hline Q07352 & ZFP36L1 & Zinc finger protein $36, \mathrm{C} 3 \mathrm{H} 1$ type-like 1 & -2.00 & $1.4 \times 10^{-4}$ & -1.03 & $5.6 \times 10^{-1}$ & 1.42 & $5.6 \times 10^{-4}$ \\
\hline P52736 & ZNF133 & Zinc finger protein 133 & -2.00 & $1.7 \times 10^{-3}$ & & & & \\
\hline Q9C009 & FOXQ1 & Forkhead box protein Q1 & & & -4.75 & $4.6 \times 10^{-5}$ & & \\
\hline
\end{tabular}


Table 2. Cont.

\begin{tabular}{|c|c|c|c|c|c|c|c|c|}
\hline \multirow{2}{*}{ Swprot } & \multirow{2}{*}{ Gene } & \multirow{2}{*}{ Protein } & \multicolumn{2}{|c|}{$3 \mathrm{dpi}$} & \multicolumn{2}{|c|}{$5 \mathrm{dpi}$} & \multicolumn{2}{|c|}{$7 \mathrm{dpi}$} \\
\hline & & & Inf/Mock F.C. & $p$-Value & Inf/Mock F.C. & $p$-Value & Inf/Mock F.C. & $p$-Value \\
\hline \multicolumn{9}{|c|}{ Down-regulated proteins } \\
\hline Q96RY5 & CRAMP1 & Protein cramped-like & & & -4.03 & $1.1 \times 10^{-3}$ & 1.01 & $7.7 \times 10^{-1}$ \\
\hline P17661 & DES & Desmin & -1.49 & $5.7 \times 10^{-3}$ & -2.63 & $1.8 \times 10^{-4}$ & 1.39 & $4.5 \times 10^{-2}$ \\
\hline P35527 & KRT9 & Keratin, type I cytoskeletal 9 & -1.55 & $1.3 \times 10^{-1}$ & -2.35 & $4.0 \times 10^{-3}$ & -2.17 & $3.4 \times 10^{-5}$ \\
\hline Q9NRM1 & ENAM & Enamelin & -1.59 & $3.2 \times 10^{-4}$ & -2.31 & $2.8 \times 10^{-4}$ & 1.51 & $3.1 \times 10^{-3}$ \\
\hline O43854 & EDIL3 & $\begin{array}{l}\text { EGF-like repeat and discoidin I-like } \\
\text { domain-containing protein } 3\end{array}$ & -1.70 & $1.1 \times 10^{-3}$ & -2.16 & $8.2 \times 10^{-4}$ & -1.33 & $2.6 \times 10^{-3}$ \\
\hline Q86YZ3 & HRNR & Hornerin & & & & & -3.94 & $1.8 \times 10^{-13}$ \\
\hline
\end{tabular}

dpi $=$ days post infection. $\mathrm{FC}=$ fold change, red $=$ significantly up-regulated , green $=$ significantly down-regulated, blue $=p$-value $<0.05$. Table is sorted first by significantly up-regulated and downregulated proteins at $3 \mathrm{dpi}$, then by those significantly down-regulated at $5 \mathrm{dpi}$; then by significantly up- and down-regulated at $7 \mathrm{dpi}$. 


\subsection{Impact of ZIKV Infection on Cellular Signaling Pathways and Function in HSerC}

All proteins and their measured quantities at 3, 5 and $7 \mathrm{dpi}$ were analyzed by Ingenuity Pathway Analysis (IPA) software to understand predicted impacts of ZIKV infection on cellular signaling pathways, bio-functions, and protein-protein networks. A total of 15, 31 and 13 Sertoli cell canonical pathways were predicted to be significantly affected at 3, 5 and 7 dpi, respectively, after ZIKV infection (Figure 3A, Figure S1A). GP6 signaling pathway was inhibited at $3 \mathrm{dpi}$, but was activated significantly at $7 \mathrm{dpi}$. Natural killer cell signaling was significantly down-regulated only at 7 dpi. Seven signaling pathways were predicted to be significantly activated at all three-time points; they are hypercytokine$\mathrm{mia} /$ hyperchemokinemia in the pathogenesis of influenza, interferon signaling, Systemic Lupus Erythematosus in B cell signaling pathway, role of PKR in interferon induction and antiviral response, neuroinflammation signaling pathway, role of pattern recognition receptors in recognition of bacteria and viruses, and NAD signaling pathway (Figure 3, Figure S1A). Interestingly, a few canonical pathways were only affected at 5 dpi, e.g., IL-17 signaling, IL-6 signaling, Cardiac hypertrophy signaling (enhanced), HIF1 $\alpha$ signaling, Glycolysis I, Gluconeogenesis I, PPAR signaling, HMGB1 signaling, Rac signaling, etc. IPA predicted the altered proteins were significantly ( $Z$ score $>1.96$ or $<-1.96$ ) associated with a total of 156 diseases and functions, which were divided into 55 for $3 \mathrm{dpi}, 95$ for $5 \mathrm{dpi}$ and 63 for 7 dpi (Table S1 Pathways associated with infectious disease were predicted to be significantly inhibited, whereas those associated with antimicrobial response, inflammatory disease, neurological disease, tissue morphology, cell death and survival, and cellular movement were the major disease and cellular function categories predicted to be activated at all three time points (Figure 3B; Figure S2). IPA also predicted a total of 801 unique upstream regulators to be activated/inhibited by ZIKV infection in Sertoli cells at $3 \mathrm{dpi}$ ( $n=400$, activated, $n=279$; inhibited 121), 5 dpi $(n=616$, activated, $\mathrm{n}=442$; inhibited 174), and 7 dpi $(n=299$, activated, $n=206$; inhibited 93), which includes cytokines, enzymes, G-protein coupled receptors, growth factors, kinases, microRNAs, peptidases, phosphatase, transcription regulators, transmembrane receptor and transporters (Table S2). The top ten predicted upstream regulators are IFNG, IFNA2, TNF, IL1B, IRF7, IFNL1, Interferon-alpha, IFR1, IRGM and STAT1 (Figure 3C). The functional analysis of the top 15 upstream regulators showed that they are associated with activation of T-lymphocytes, leukocytes, cells, recruitment of leukocytes, antiviral response, and RNA virus replication (Figure S1B).

IPA also built protein-protein networks of the significantly affected proteins based on their direct or indirect interactions. There were 12, 9, and 10 protein-protein interaction networks (Score $>20$, Molecules $>13$ ) predicted by IPA analysis of the altered proteins at 3, 5 and $7 \mathrm{dpi}$, respectively. The predicted networks were associated with different cellular functions and diseases, including cell morphology, cellular assembly and organization, neurological disease, lipid metabolism, molecular transport, cardiovascular disease, connective tissue disorders, antimicrobial response, cell cycle, and endocrine system development (Table S3). Cancer, connective tissue disorders, organismal injury and abnormalities (Score 38, Focus Molecules 23) were the most affected predicted proteinprotein networks at $3 \mathrm{dpi}$, cardiovascular disease, cell death and survival, and connective tissue disorders (Score 42, Focus Molecules 25) were the most affected at $5 \mathrm{dpi}$, and antimicrobial response, and infectious diseases, inflammatory response (Score 41, Focus Molecules 22) were predicted to be the most affected at $7 \mathrm{dpi}$ (Figure 3D; Figure S2C,D). 
A

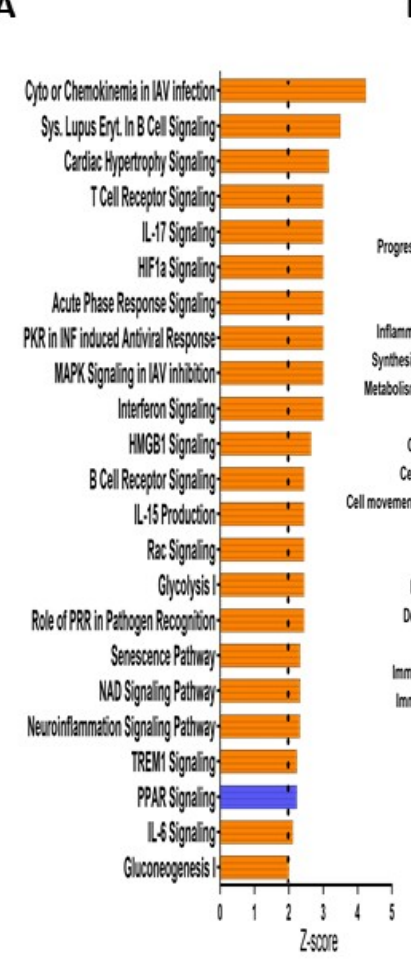

B

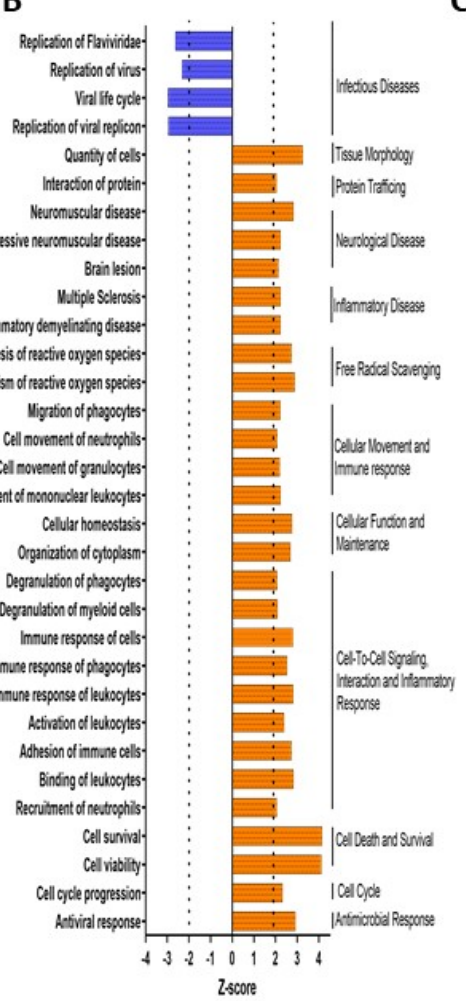

C
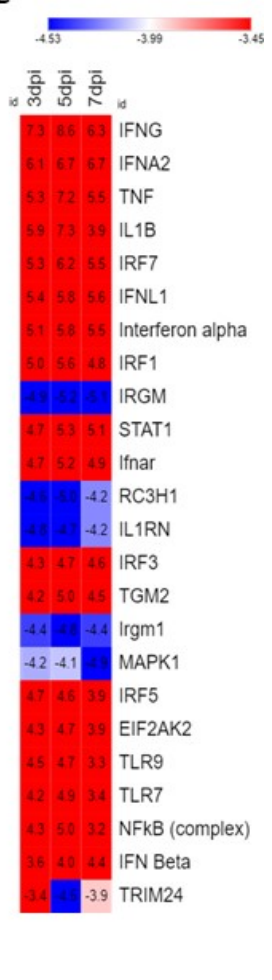

D

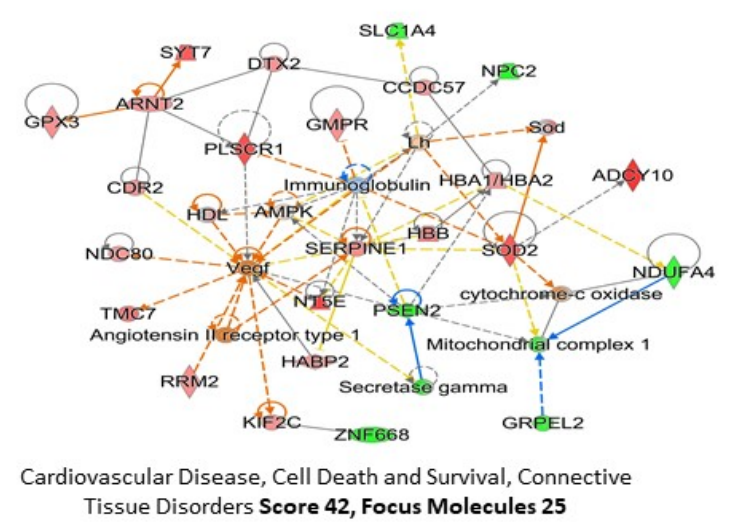

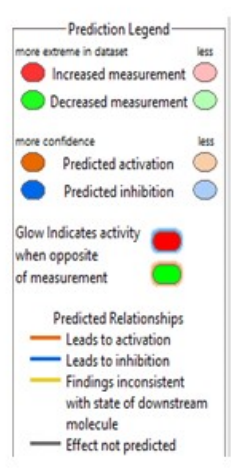

Figure 3. IPA-predicted activation and inhibition of bio-functions, canonical pathways, proteinprotein networks, and upstream molecules after ZIKV infection in HSerC. (A) Top-most canonical pathways predicted to be significantly activated or inhibited by IPA at 5 days post ZIKV infection. (B) Top bio-functions and predicted activation or inhibition Z-scores indicated at 5 dpi. Activation is indicated by a positive Z-score and the inhibited bio-functions are indicated by negative Z-scores. (C) The top 25 upstream regulators, predicted to be affected at 3, 5 and 7 dpi after ZIKV infection in HSerC. Red indicates up-regulation, blue indicates down-regulation. Numbers in boxes show the significance of alteration measured by Z-score. (D) The most affected protein networks at 5 dpi by ZIKV infection in HSerC. Red and green represent up-regulation and down-regulation, respectively; gray proteins denote that they were recognized in the present study, but not significantly regulated; colorless proteins interact with molecules in the network, but were not identified in our study. Abbreviations. dpi $=$ Days post infection.

\subsection{HSerC Activates Immune Response against ZIKV Infection}

To understand the impact of ZIKV infections across the three time points, we selected the list of 50 commonly affected proteins (Figure 4A,B) and performed IPA core analysis. This analysis showed that the most affected proteins were predicted to be associated with canonical pathways involving replication of flavivirus, replication of RNA virus and in- 
terferon signaling pathways (Figure 4C). The infectious disease, inflammatory response, neurological disease, and antimicrobial disease responses were the most affected disease and functions predicted by heatmap analysis of IPA (Figure 4D). The protein-protein network analysis predicted their links with cell-to-cell signaling and interaction, infectious diseases, and post-translational modification (Figure $4 \mathrm{E}$ ).
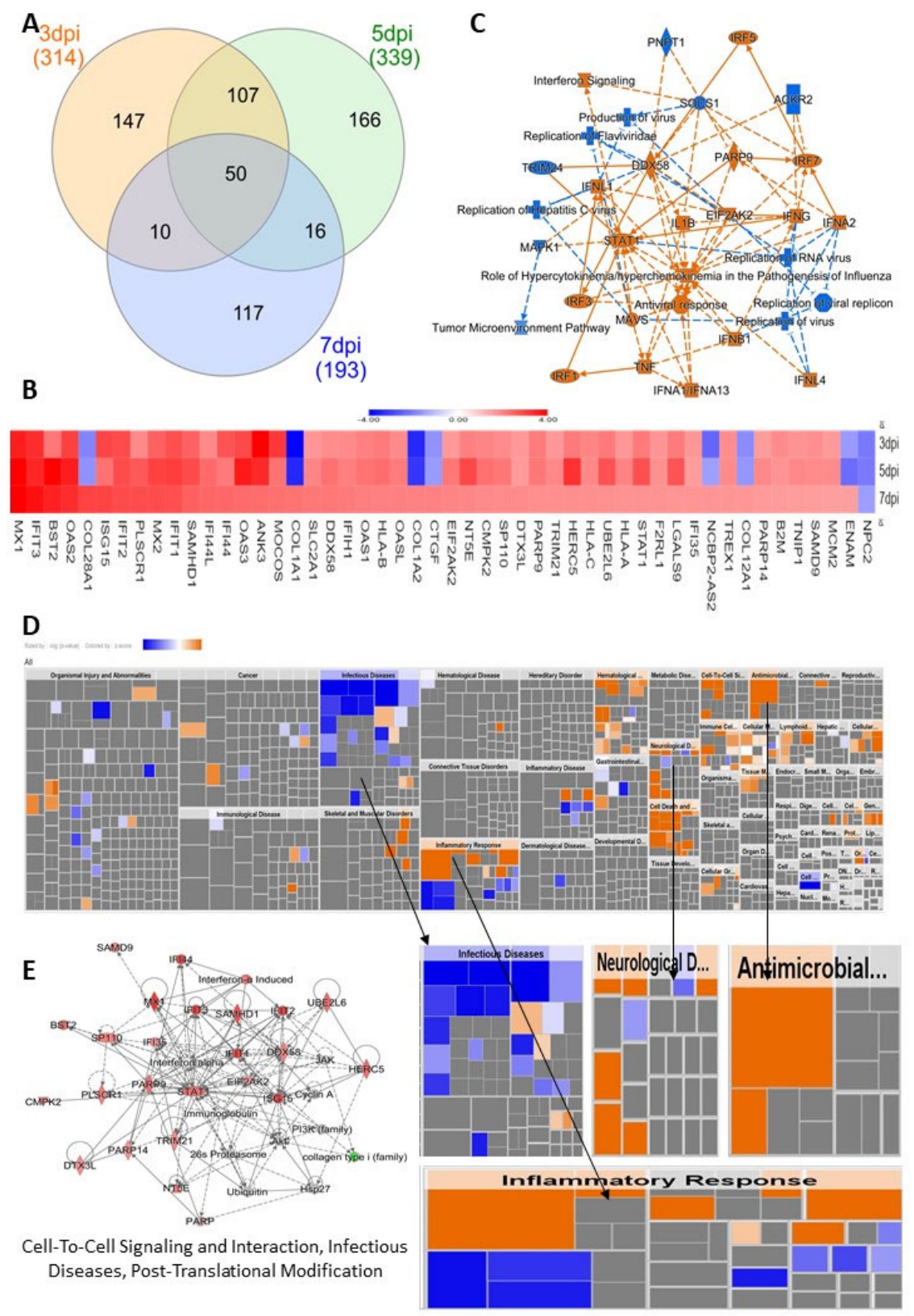

Figure 4. Impact of commonly affected proteins in HSerC by ZIKV on cellular functions and diseases. (A) Venn diagram of altered proteins at 3, 5 and 7 dpi. (B) Summary of IPA analysis showing the most affected bio-functions, canonical pathways and affected protein networks and their relationship by the commonly affected proteins. (C) Heatmap of the 50 commonly affected proteins at 3, 5 and $7 \mathrm{dpi}$. Red/orange and blue represent up-regulation and down-regulation, respectively. (D) Heat map of the disease and biofunctions affected by the commonly affected proteins. (E) The proteinprotein interaction networks affected by the commonly altered proteins. Red and green represent up-regulation and down-regulation, respectively; gray proteins denote that they were recognized in the present study, but not significantly regulated; colorless proteins interact with molecules in the network, but were not identified in our study. Abbreviations. dpi = Days post infection. 
The immune response of antigen-presenting cells, immune response of phagocytes and leukocytes, activation of antiviral response, phagocytosis of cells and innate immune response also were predicted to be activated at 3,5 and 7 days post-ZIKV infection (Figure 5A). Several proteins involved in immune response regulating signaling pathways were also significantly stimulated by ZIKV infection, which includes interferon signaling, PKR interferon induction and antiviral response, role of pattern recognition receptor in recognition (PRRR) of bacteria and virus, Th1 pathway, HMGB1, TREM1, IL-17, IL-8, IL-6, IL-15 signaling, etc. (Figure 5B). Across the three time points after ZIKV infection, interferon signaling was one of the most affected signaling pathways in Sertoli cells. ZIKV caused significant alteration of 12 protein levels that are predicted to regulate interferon signaling pathways (Figure 5C). Based on the expression values of these proteins, IPA predicted a significant activation of the pathways at $3 \mathrm{dpi}$ (Z-score 2.82), $5 \mathrm{dpi}$ (Z-score 3.0), and $7 \mathrm{dpi}$ (Z-score 3.0) (Figure 5D).

A

(

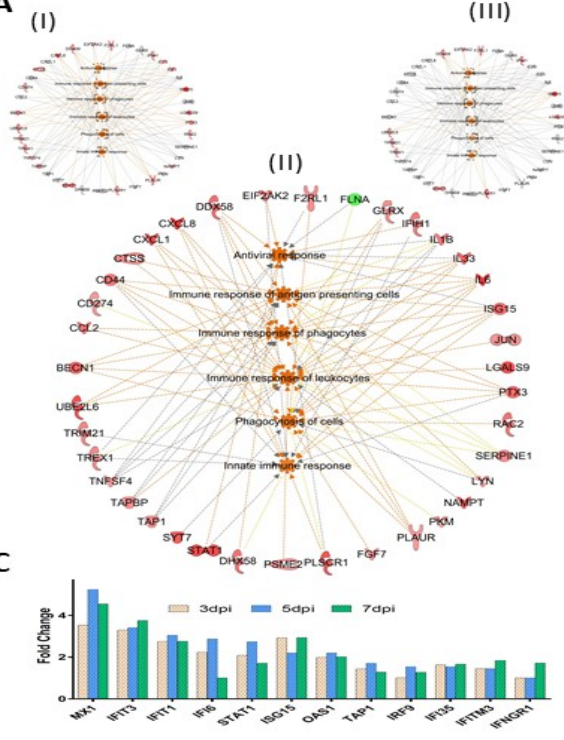

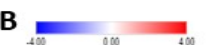

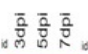

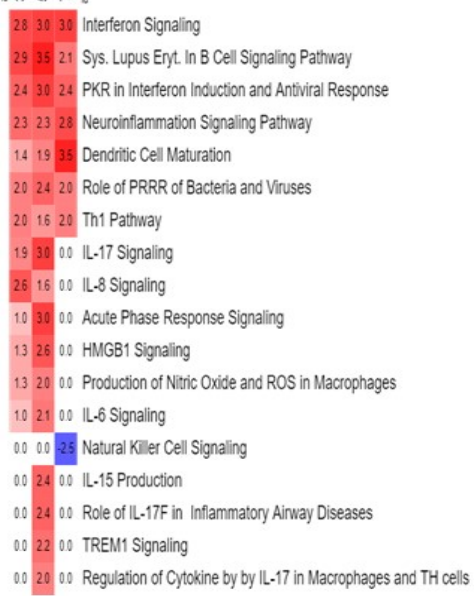

0020 00 Regulation of Cytokine by by IL-17 in Macrophages and TH cells

D
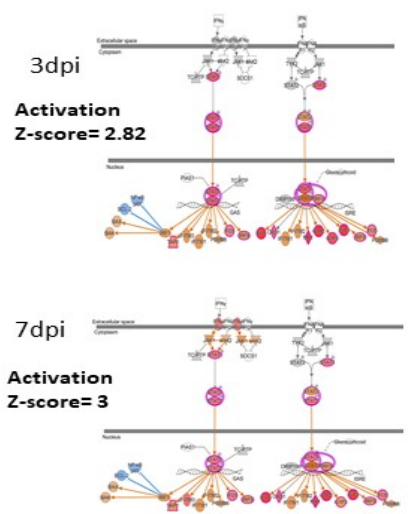

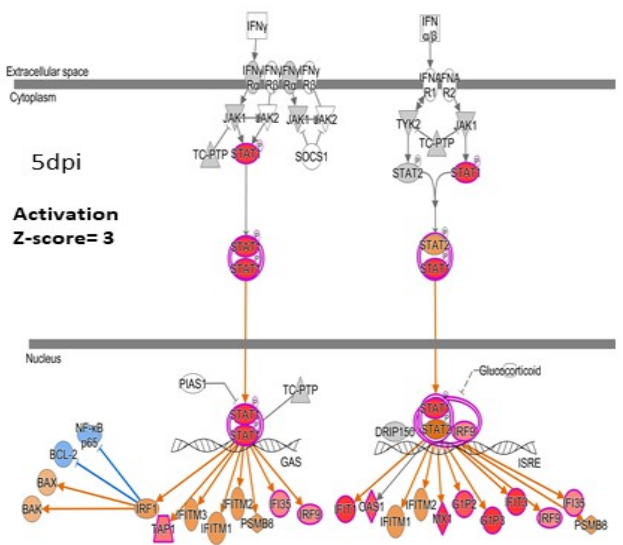

Figure 5. Immune response in HSerC after ZIKV infection. (A) Bio-functions related to immune response pathways affected at (I) $3 \mathrm{dpi}$, (II) $5 \mathrm{dpi}$, and (III) $7 \mathrm{dpi}$. (B) Canonical pathways associated with immune response pathways affected by ZIKV infection in Sertoli cells. Red and blue represent up-regulation and down-regulation, respectively. The number in each box shows the activation Z-score predicted by IPA. (C) Proteins in Interferon signaling pathways affected by ZIKV infection. (D) Activation of Interferon signaling pathway by ZIKV infection. Red and green represent upregulation and down-regulation, respectively; gray proteins denote that they were recognized in the present study, but not significantly regulated; colorless proteins interact with molecules in the network, but were not identified in our study. Abbreviations. dpi = Days post infection. 
We identified several proteins associated with establishment of viral infection and replication that were significantly affected by ZIKV infection in HSerC (Figure 6A). Based on their expressions, IPA predicted that proteins associated with Flaviviridae infection and replication were significantly inhibited (Figure 6B). Interestingly, these analyses also predicted that proteins associated with replication of some other viruses such as Hepatitis C virus, Herpesviridae, murine herpesvirus 4, Orthomyxoviridae, and coronavirus were affected (Figure 6B). These proteins associated with viral infection and replication are predicted to be found in the cytoplasm, nucleus, cell membrane, and extracellular space of Sertoli cells (Figure 6C).

A

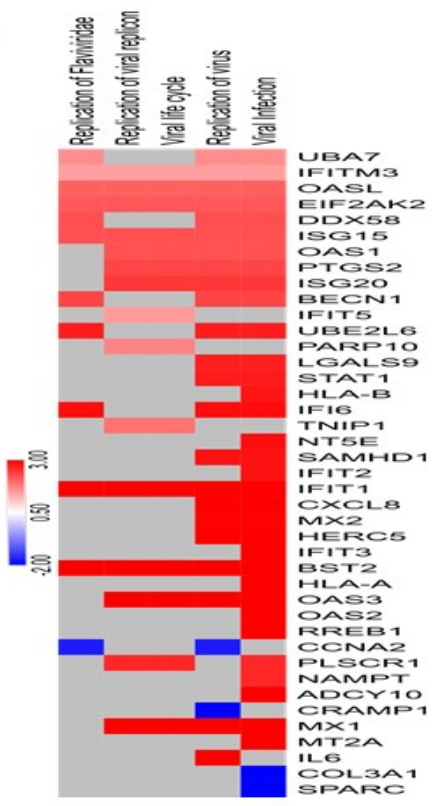

B

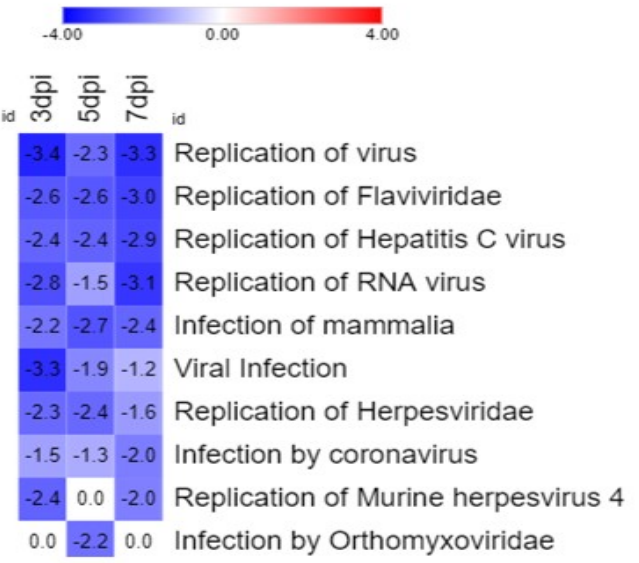

C

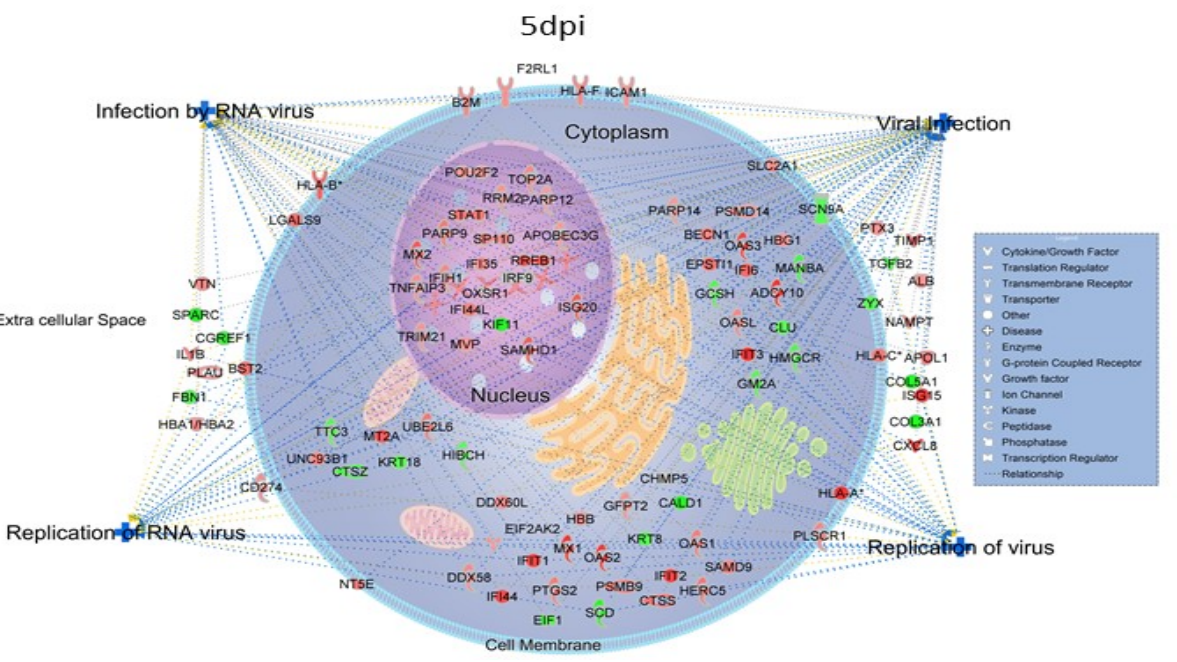

Figure 6. Viral replication was suppressed by the cellular response in HSerC after ZIKV infection. (A). Heat map of the proteins involved in virus infection, lifecycle and replication at $5 \mathrm{dpi}$. Red and blue represent up-regulation and down-regulation of protein expression, respectively. (B) Replication of different family of viruses were predicted to be inhibited by the significantly affected proteins at 3 , 5 and $7 \mathrm{dpi}$. The number in the box indicates the Z-score of the representative function or disease. (C) Cellular localization of the altered proteins predicted to be involved in the regulation of viral infection and in infection by, and replication of, RNA viruses. Red and green represent up-regulation and down-regulation of protein expression, respectively. Abbreviations. dpi = Days post infection. 


\subsection{ZIKV Infection Impacts Carbohydrate Metabolism in Sertoli Cells}

At 5 dpi ZIKV caused significant activation of glycolysis $(Z$-score $=2.44)$ and gluconeogenesis $(Z$-score $=2.0)$ pathways (Figure $3 \mathrm{~A}$ ). There were 18 Sertoli cell proteins associated with the glycolysis and gluconeogenesis pathway that were significantly affected by ZIKV infection. Eleven of their levels (SLC2A1, IL1B, PRP14, FABP1, ADCY10, PGK1, IL6, ENO2, ENO1, PGAM1 and SL39A14) were altered (Figure 7A) at 5 dpi and predicted to significantly activate $(\mathrm{Z}$ score $=2.44)$ the glycolysis pathway (Figure 7B). ZIKV infection significantly affected 20,19 and 11 proteins related to carbohydrate metabolism and cellular energy production pathways at 3,5 and $7 \mathrm{dpi}$, respectively (Figure 7C; Figure S3). These proteins are associated with the synthesis of ATP and its concentration in cells, metabolism of carbohydrate, phosphatidic acid, D-glucose, D-hexose, monosaccharide, phospholipids and synthesis of carbohydrate and phospholipid.

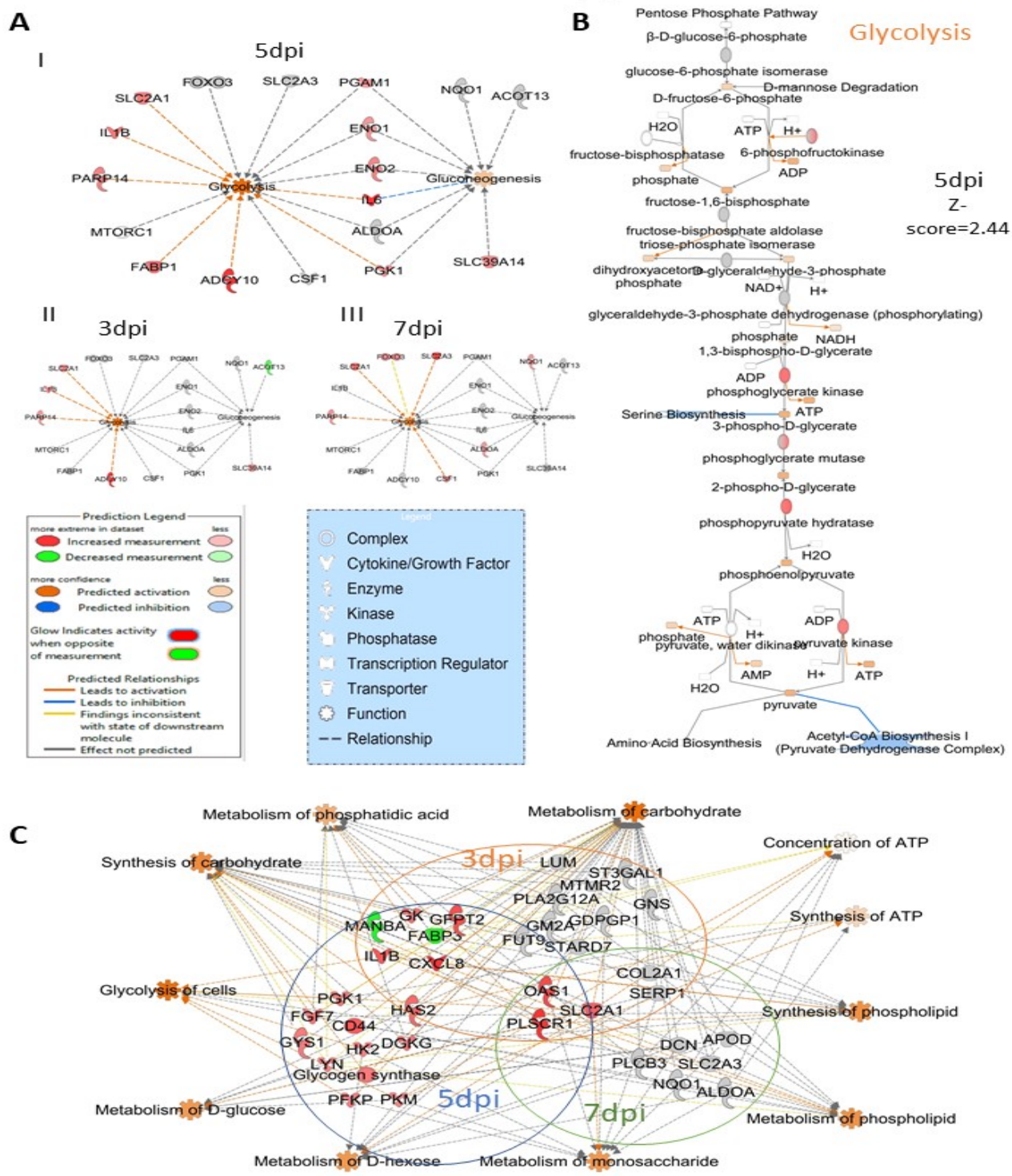

Figure 7. ZIKV infection impacts carbohydrate metabolism in HSerC. (A) Proteins and enzymes involved in glycolysis and gluconeogenesis pathways affected by ZIKV infection at I. 5 dpi, II. 3 dpi and III. 7 dpi. IPA predicted the impact of ZIKV infection on (B) glycolysis pathway at 5 dpi in Sertoli cells. (C) Proteins involved in carbohydrate metabolism and energy production in cells affected by ZIKV infection. Red and green represent up-regulation and down-regulation of protein levels, respectively. Abbreviations. Dpi $=$ Days post infection. 


\subsection{ZIKV Infection Significantly Affects Proteins That May Increase Cardiovascular Disease}

ZIKV infection significantly affected 20 proteins (Figure 8D) involved in the cardiac hypertrophy pathway and significantly activated $(Z$-score $=3.1)$ proteins within the pathway at $5 \mathrm{dpi}$ (Figure $8 \mathrm{~A}$ ). However, at $3 \mathrm{dpi}$ and $7 \mathrm{dpi}$, the proteins in this pathway were not significantly impacted by ZIKV infection (Figure 8B,C). IPA has a database of 617 proteins associated with the increase of cardiovascular disease. Among these, 23, 28 and 14 proteins were significantly affected by 3,5 and 7 days post-ZIKV infection, respectively. The significantly altered proteins are associated with atherosclerosis, ventricular dysfunction, myocardial dysfunction, cardiac lesion, hypertrophy of heart, morphology of heart and cardiovascular system and dysfunction of heart (Figure 8E-G).

A
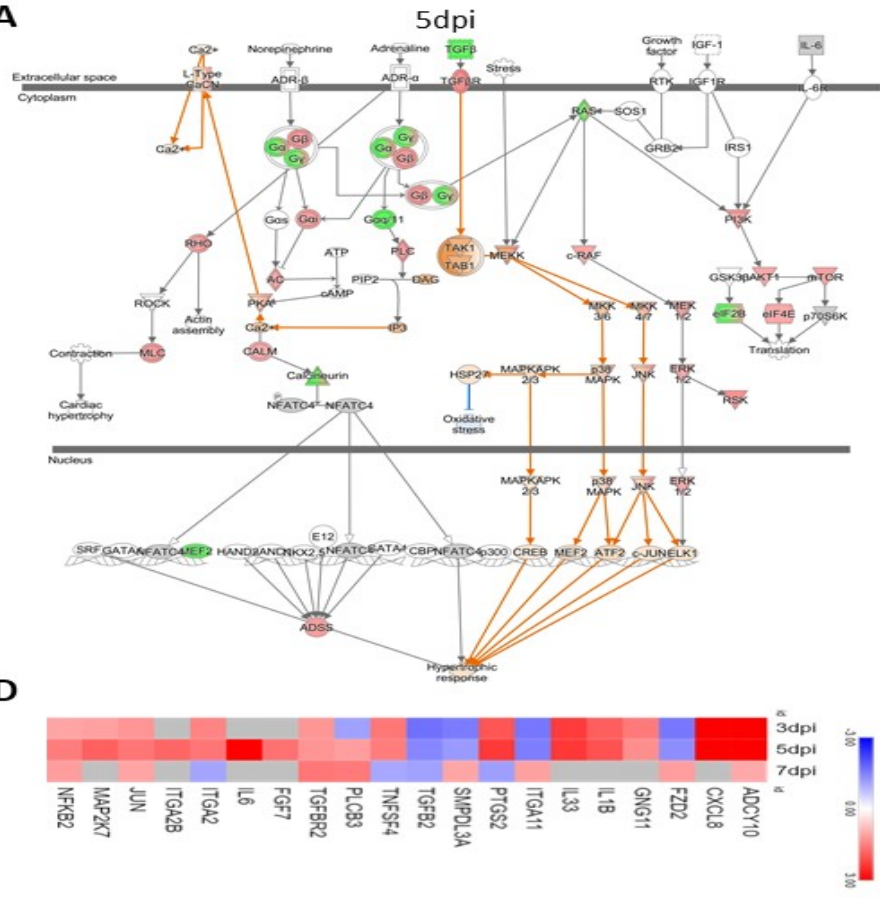

E

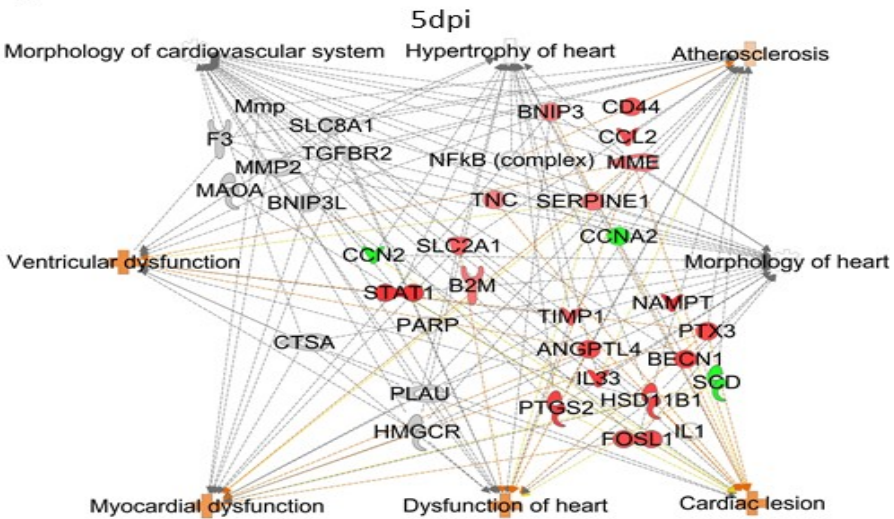

B $\quad 3 d p i$
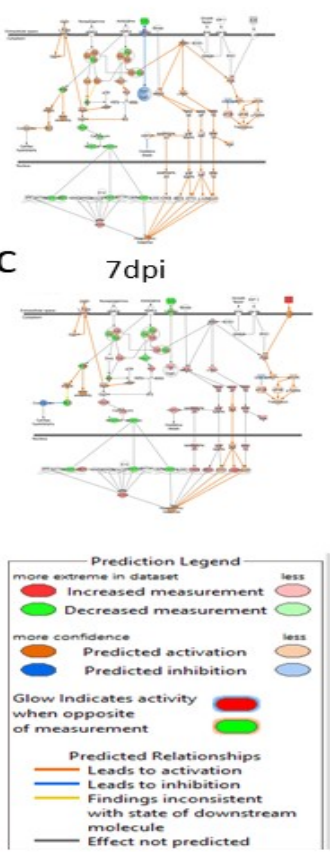

$\mathbf{F}$

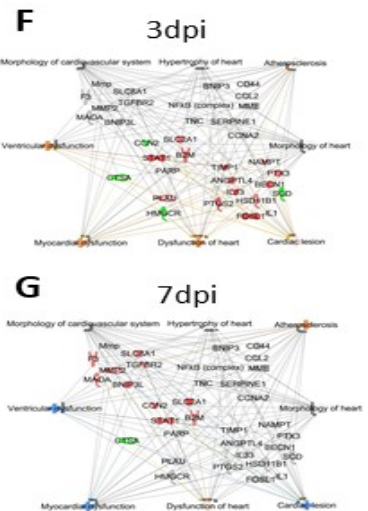

Figure 8. ZIKV infection significantly affects the levels of proteins involved in the cardiac hypertrophy pathway. IPA predicted activation of the cardiac hypertrophy pathway by Zika virus infection by (A) $3 \mathrm{dpi}$, (B) 7 dpi and (C) 5 dpi. (D). Heatmap of the proteins involved in cardiac hypertrophy pathway altered by ZIKV infection in HSerC. In the heatmap, red and blue represent up-regulation and down-regulation, respectively. Gray indicates the proteins were not detected at the respective time point. HSerC proteins associated with pathways that increase cardiovascular disease, that are affected by ZIKV infection at (E) 5 dpi (F) 3 dpi and (G) 7 dpi. Red and green represent up-regulation and down-regulation of protein expression, respectively. Abbreviations. dpi = Days post infection. 


\subsection{Validation of Mass Spec Data by Western Blot}

Five proteins (CLIC1, SPARC, STAT1, STAT3 and PSMA2) were selected for validation based on their expression fold-change and availability of commercial antibodies. Western blot was done (Figure 9A), and the intensities of protein bands were measured to determine the fold difference between the infected and mock conditions. The fold change of each protein measured by Western blot at three different time points was compared side-by-side to the expression values determined by mass spectrometry (Figure 9B). All five proteins followed the same trend of expression by Western blot as measured by TMT-mass spectrometry.

A

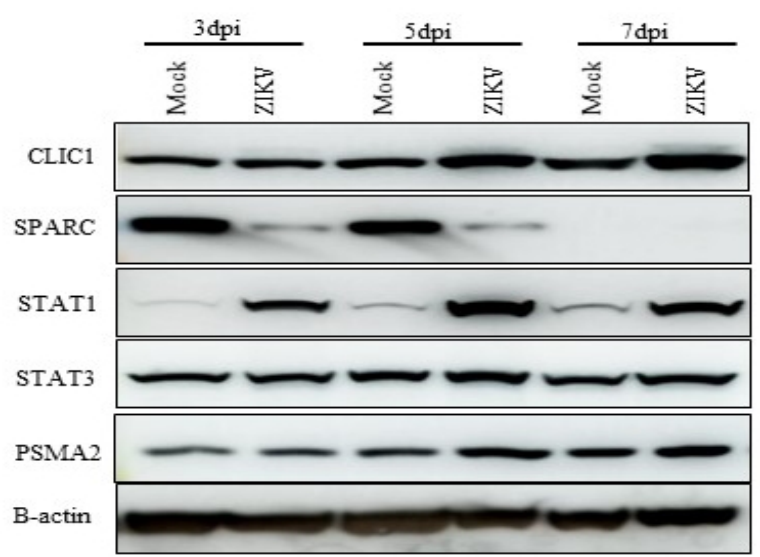

B

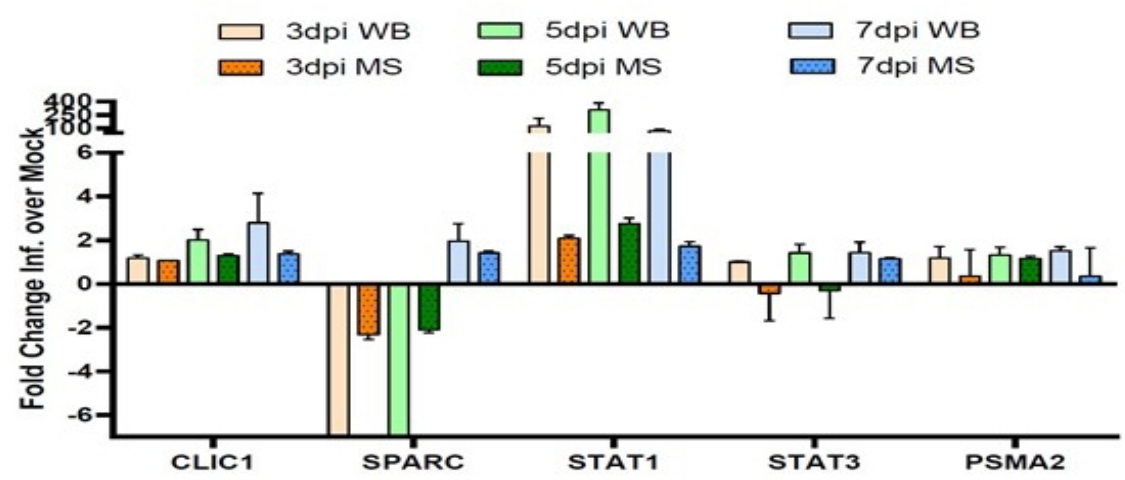

Figure 9. Western blot validation of proteomic changes detected by mass spectrometry (MS) analysis. Sertoli cell lysates were collected after ZIKV infection (MOI:3) at 3, 5 and $7 \mathrm{dpi}$. In total, $20 \mu \mathrm{g}$ of proteins were separated by SDS-PAGE gel electrophoresis. (A) Expression of CLIC1, SPARC, STAT1, STAT3 and PSMA2 were detected by Western blot using specific antibodies. (B) Expression values of the proteins from Western-blot were quantified by ImageJ V1.8.0 from three replicates and plotted side-by-side to MS expression values. Expression of protein by Western blot was normalized to B-actin expression. Abbreviations: $\mathrm{dpi}=$ Days post infection; $\mathrm{WB}=$ Western blot; $\mathrm{MS}=$ Mass spectrometry; ZIKV = Zika virus.

\section{Discussion}

The mechanism(s) underlining the long-term persistence of ZIKV in male semen is not yet clearly understood. The sexual transmission of the virus raises the risk of initiating outbreaks in non-endemic regions, even in the absence of a mosquito vector. Furthermore, the pathologic consequences of viral persistence in the male genital tract are unclear. In our previous study, to investigate ZIKV infection-induced HSerC proteome alterations, we applied SOMAscan, a multi-plexed targeted technology that can identify 
over 1300 proteins from each sample [36]. Although SOMAscan, as a targeted approach, can identify many non-abundant proteins, it is still limited in its detection limit to the specified 1305 proteins [42]. Therefore, in this study, we used a six-plex TMT-based mass spectrometry approach to determine additional HSerC proteome alterations to provide a broader insight on the impact of ZIKV infection. However, unlike the previous study, we excluded the $1 \mathrm{dpi}$ time point due to a small number of significantly affected proteins [36] and included $7 \mathrm{dpi}$ to analyze the impact of ZIKV on Sertoli cells at a later stage. In addition, as previously reported [36,37,43], Zika virus infection had no apparent cytopathic impact on Sertoli cells after infection (Figure 1A). However, ZIKV replication [36] and viral protein expression (Figure 1B,C) were significantly reduced at 7 dpi in Sertoli cells, but infectious virus has been detected for at least 6 weeks in these cells [37]. This indicates that ZIKV initially undergoes a robust infection in Sertoli cells, but it is subsequently controlled, likely by cellular immune responses and later it maintains a low level of persistence. In this study, we have seen that most of the significantly affected proteins were down-regulated at $3 \mathrm{dpi}$, and up-regulated at $5 \mathrm{dpi}$ as observed before [36]. This confirms a consistent time-dependent switch of proteomic alteration caused by ZIKV infection in Sertoli cells. Among the most affected proteins, FBXO11 has been associated with Neurodevelopmental disorders, mental retardation, and autism [44,45]. However, KIF1A expression anomaly was linked with neurological disorders in children [46]. Therefore, the alteration of FBXO11 and KIF1A could be associated with microcephaly and Guillain-Barré syndrome that develop during and/or after ZIKV infection. UHRF2 is one of the central regulators of cell cycle machinery [47]. TYR is responsible for initiating the conversion of tyrosine to melanin, which is responsible for pigmentation of eye, skin and hair [48]. Abnormalities in TYR expression may cause albinism $[49,50]$. FOXQ1 is a transcription regulator that controls the cell cycle, cell proliferation and is associated with cancers [51-54], and that inhibits macrophage recruitment [55], and activates Wnt signaling [56].

\subsection{HSerC Activates Immune Response against ZIKV Infection}

The human testis maintains an immune-privileged environment, separated from the body cavity by the blood-testis barrier (BTB), made with Sertoli cells [57]. However, to combat invading microbial pathogens, the testis possesses a local immune defense system [28]. In response to ZIKV infection, HSerC induced changes in the levels of proteins predicted to activate a strong immune response at 3,5 and $7 \mathrm{dpi}$. According to the analysis of the 50 predominantly affected proteins, the inflammatory and antimicrobial response pathways were significantly triggered after ZIKV infection of HSerC (Figure 4D). The protein-protein interaction analysis also predicted antimicrobial response, infectious diseases, and inflammatory response as activated across the three-time points (Table S3). In addition, at least 17 cellular signaling pathways associated with immune response were predicted to be significantly activated in HSerC after ZIKV infection (Figure 5B). In agreement with our findings, a previous study based on transcriptomic analysis [35] also determined that interferon signaling, IL-15, role of PKR interferon induction and antiviral response, HMGB1 and role of pattern recognition receptor in recognition of bacteria and virus were significantly affected by ZIKV infection of Sertoli cells. Further analysis of the significantly affected proteins (Figure 5A) and upstream molecules (Figure S1B) indicated that proteins involved in immune response of antigen-presenting cell pathways (APCs), phagocytes, leukocytes and T lymphocytes were significantly activated. Sertoli cells' potent immune response may have inhibited viral replication, as anticipated by IPA (Figure 6B), also seen by the decreased quantity of viral proteins at 5 and $7 \mathrm{dpi}$ (Figure 1B,C). Interestingly, proteins associated with antiviral response against Flaviviridae may significantly suppress other human viruses such as Hepatitis C virus, Herpesvirus, and Coronavirus (Figure 6A,B). This suggests that targeting commonly used host proteins or pathways might lead to the development of a broad-spectrum antiviral therapy in the future. 


\subsection{ZIKV Affects Proteins Involved in Carbohydrate Metabolism}

Viruses use biomolecules from the host and induce anabolism to create the macromolecules needed for virion replication and assembly. As a result, it is no surprise that viral infection forces host cells to modify their metabolism in order to support effective virus replication [58]. Many oncogenic viruses use the glycolysis pathway, such as human papillomavirus (HPV), hepatitis $\mathrm{C}$ virus (HCV), hepatitis B virus (HBV), Kaposi's sarcoma-associated herpesvirus (KSHV), Epstein-Barr virus (EBV), Merkel cell polyomavirus (MCPyV), and Adenovirus [59,60]. However, several non-oncogenic viruses were also found to activate the glycolysis pathways, including Herpes simplex virus 1 and 2 [61], and Human cytomegalovirus [58]. Dengue virus (DENV), another relative of ZIKV, depends on the glycolysis pathways for its replication [62]. High concentrations of glucose can restrict the growth of ZIKV in human kidney cells [63]. ZIKV infection increases glucose incorporation into the TCA cycle in mosquito cells, but the mechanism is not clear [64]. In HSerC, proteins involved in glycolysis and gluconeogenesis pathways were significantly activated by ZIKV infection (Figures $3 \mathrm{~A}$ and 7B). In addition, the metabolism of D-fructose, monosaccharides, phosphatidic acid, phospholipids were also predicted to be significantly activated by the significantly altered proteins (Figure 7C, Figure S3). Previously, we detected PGAM1, a key regulator of glycolysis and gluconeogenesis pathways, as significantly up-regulated by ZIKV infection in HSerC [36]. However, proteins involved in energy generation in Sertoli cells by lactate and lipid oxidization through the Peroxisome Proliferator-Activated Receptor (PPAR) signaling pathway [65] were significantly down-regulated by ZIKV infection (Figure 3A), as reported previously [36]. These results suggest that ZIKV hijacks carbohydrate metabolism to usurp cellular energy generation in HSerC. The glycolysis pathway could be a potential target against ZIKV and needs further investigation in the future.

\subsection{ZIKV Infection Affects Proteins Associated with an Increase in Cardiovascular Disease}

Surprisingly, we found many proteins involved in cardiac disease that were significantly affected (Figure 8E-G) during ZIKV infection of HSerC. These proteins are associated with significant activation of atherosclerosis, ventricular dysfunction, myocardial dysfunction, and cardiac lesion (Figure 8E-G). RGS5 is one of the topmost affected proteins detected in HSerC after ZIKV infection. It is a GTPase activator that protects against cardiac hypertrophy and cardiac fibrosis [66]. Cardiovascular disease was a frequently detected protein-protein network predicted by IPA at all three-time points after ZIKV infection (Table S3). In addition, levels of at least 20 proteins involved in the cardiac hypertrophy signaling pathway were significantly altered by ZIKV, causing significant predicted activation of the pathway (Figures $3 \mathrm{~A}$ and $8 \mathrm{~A}$ ) at $5 \mathrm{dpi}$ in HSerC. Thus far, cardiovascular complications have been associated with ZIKV infection by a case report [67], an observational study [68], and in an animal study [69]. Although identified by us in HSerC, the cardiac hypertrophy signaling pathway could be a possible mechanism of cardiac complications in ZIKV-infected patients. However, the current study has limitations, as it was performed in a non-cardiac cell line. To fully comprehend the link between ZIKV and cardiovascular abnormalities and its mechanism, extensive investigation is necessary.

\section{Conclusions}

Understanding the mechanisms of ZIKV persistence in the male genital tract is critical for treatment of the disease, antiviral/vaccine development and overall control of viral transmission. Multiple studies have suggested that Sertoli cells could be a potential reservoir for this viral persistence. Sertoli cells build the blood-testis barrier that maintains an isolated environment for optimal development of the germ cells and provides sufficient nutrients and signals for sequential differentiation into sperm by spermatogenesis [30]. ZIKV infection of Sertoli cells may impact sperm development and male fertility. Moreover, a clear understanding of the replication dynamics of the virus in an immune-privileged environment is necessary for antiviral or vaccine development. Previous studies of ZIKV-infected 
Sertoli cells have reported the roles of a particular protein [70] or its impact on the BTB [71]. However, this is the first study that uses mass spectrometry to understand global proteomic alterations induced by ZIKV infection in HSerC at 3, 5 and 7 dpi. Longitudinal proteome analysis facilitates the identification of critical proteins and pathways for the successful ZIKV infection cycle and provides detailed knowledge that may be used in therapeutic intervention. Many host proteins and signaling pathways crucial for ZIKV replication were found in this study, and these could be targeted for antiviral or diagnostic tool development in the future.

Supplementary Materials: The following supporting information can be downloaded at: https: / / www.mdpi.com/article/10.3390/v14020377/s1, Supplementary Table S1. Disease and functions affected by ZIKV infection in HSerC. Supplementary Table S2. IPA predicted the upstream regulators affected by ZIKV infection in Sertoli cells. Supplementary Table S3. The protein-protein networks affected by ZIKV infection at 3,5 and 7 dpi in HSerC. Supplementary Figure S1. (A) Comparison of canonical pathways affected at 3,5 and 7 dpi during ZIKV infection in HSerC. Red indicates upregulation, blue indicates down-regulation. Numbers in the boxes show the significance of alteration measured by Z-score. (B) Top 15 upstream regulators associated with cellular function and disease. Supplementary Figure S2. IPA-predicted activation and inhibition of bio-functions, and proteinprotein network at 3 and $7 \mathrm{dpi}$ in HSerC. Top bio-functions and their predicted activation or inhibition Z-scores indicated at (A) 3 and (B) $7 \mathrm{dpi}$. Activation is indicated by positive Z-Score and the inhibited bio-functions are indicated by negative Z-scores. The most effected protein-protein networks at (C) 3 dpi and (D) 7 dpi by ZIKV infection in HSerC. Red and green represent up-regulation and down-regulation, respectively; gray proteins denote that they were recognized in the present study, but not significantly regulated; colorless proteins interact with molecules in the network, but were not identified in our study. Abbreviations: dpi = Days post infection. Supplementary Figure S3: Proteins involved in the metabolism of carbohydrate and energy production in cells affected by ZIKV infection at $3 \mathrm{dpi}$ and $7 \mathrm{dpi}$. Red and green represent up-regulation and down-regulation, respectively; gray proteins denote that they were recognized in the present study, but not significantly regulated; colorless proteins interact with molecules in the network, but were not identified in our study. Abbreviations: $\mathrm{dpi}=$ Days post infection.

Author Contributions: Conceptualization, M.-u.R. and K.M.C.; methodology, Y.L.; software, M.-u.R.; validation, V.S., formal analysis, M.-u.R. and K.M.C.; investigation, M.-u.R. and K.M.C.; writingoriginal draft preparation, M.-u.R.; writing-review and editing, M.-u.R. and K.M.C., visualization, M.-u.R. and K.M.C.; supervision, K.M.C.; project administration, K.M.C.; funding acquisition, K.M.C. All authors have read and agreed to the published version of the manuscript.

Funding: This study was funded by grant MOP-106713 from the Canadian Institutes of Health Research to KMC. MuR was supported by a Research Manitoba Studentship.

Institutional Review Board Statement: Not applicable.

Informed Consent Statement: Not applicable.

Data Availability Statement: Spectra (in MGF format) and an overall log2 protein expression matrix are available at the University of California, San Diego's MassIVE archive (massive.ucsd.edu) under the accession MSV000088634.

Conflicts of Interest: The authors declare they have no conflicts of interest with this study.

\section{References}

1. Dick, G.W.A.; Kitchen, S.F.; Haddow, A.J. Zika Virus (I). Isolations and serological specificity. Trans. R. Soc. Trop. Med. Hyg. 1952, 46, 509-520. [CrossRef]

2. Shuaib, W.; Stanazai, H.; Abazid, A.G.; Mattar, A.A. Re-Emergence of Zika Virus: A Review on Pathogenesis, Clinical Manifestations, Diagnosis, Treatment, and Prevention. Am. J. Med. 2016, 129, 879.e7-879.e12. [CrossRef] [PubMed]

3. Petersen, L.R.; Jamieson, D.J.; Powers, A.M.; Honein, M.A. Zika Virus. N. Eng. J. Med. 2016, 374, 1552-1563. [CrossRef] [PubMed]

4. Ferraris, P.; Yssel, H.; Missé, D. Zika virus infection: An update. Microbes Infect. 2019, 21, 353-360. [CrossRef]

5. Lessler, J.; Chaisson, L.H.; Kucirka, L.M.; Bi, Q.; Grantz, K.; Salje, H.; Carcelen, A.C.; Ott, C.T.; Sheffield, J.S.; Ferguson, N.M.; et al. Assessing the global threat from Zika virus. Science 2016, 353, 6300. [CrossRef] 
6. $\quad$ Ribeiro, L.S.; Marques, R.E.; de Jesus, A.M.R.; de Almeida, R.P.; Teixeira, M.M. Zika crisis in Brazil: Challenges in research and development. Curr. Opin. Virol. 2016, 18, 76-81. [CrossRef]

7. Baud, D.; Gubler, D.J.; Schaub, B.; Lanteri, M.C.; Musso, D. An update on Zika virus infection. Lancet 2017, 390, $2099-2109$. [CrossRef]

8. WHO. Zika Epidemiology Update. 2019. Available online: https://www.who.int/emergencies/diseases/zika/zikaepidemiology-update-july-2019.pdf (accessed on 24 December 2021).

9. Faizan, M.I.; Abdullah, M.; Ali, S.; Naqvi, I.H.; Ahmed, A.; Parveen, S. Zika Virus-Induced Microcephaly and Its Possible Molecular Mechanism. Intervirology 2016, 59, 152-158. [CrossRef]

10. Shang, P.; Zhu, M.; Wang, Y.; Zheng, X.; Wu, X.; Zhu, J.; Feng, J.; Zhang, H.L. Axonal variants of Guillain-Barré syndrome: An update. J. Neurol. 2021, 268, 2402-2419. [CrossRef]

11. Brasil, P.; Sequeira, P.C.; Freitas, A.D.A.; Zogbi, H.E.; Calvet, G.A.; de Souza, R.V.; Siqueira, A.M.; de Mendonca, M.C.L.; Nogueira, R.M.R.; de Filippis, A.M.B.; et al. Guillain-Barré syndrome associated with Zika virus infection. Lancet 2016, 387, 1482. [CrossRef]

12. WHO. Zika Situation Report. 2016. Available online: http://www.who.int/emergencies/zika-virus/situation-report/25-august2016/en (accessed on 25 August 2017).

13. Faye, O.; Freire, C.C.; Iamarino, A.; Faye, O.; de Oliveira, J.V.C.; Diallo, M.; Zanotto, P.M.; Sall, A.A. Molecular Evolution of Zika Virus during Its Emergence in the 20th Century. PLoS Negl. Trop. Dis. 2014, 8, 36. [CrossRef]

14. Turmel, J.M.; Abgueguen, P.; Hubert, B.; Vandamme, Y.M.; Maquart, M.; Le Guillou-Guillemette, H.; Leparc-Goffart, I. Late sexual transmission of Zika virus related to persistence in the semen. Lancet 2016, 387, 2501. [CrossRef]

15. Hastings, A.K.; Fikrig, E. Focus: Infectious Diseases: Zika Virus and Sexual Transmission: A New Route of Transmission for Mosquito-borne Flaviviruses. Yale J. Biol. Med. 2017, 90, 325. Available online: https://www.ncbi.nlm.nih.gov/pmc/articles/ PMC5482308/ (accessed on 1 January 2022).

16. Moreira, J.; Peixoto, T.M.; Siqueira, A.M.; Lamas, C.C. Sexually acquired Zika virus: A systematic review. Clin. Microbiol. Infect. 2017, 23, 296-305. [CrossRef]

17. Bujan, L.; Mansuy, J.M.; Hamdi, S.; Pasquier, C.; Joguet, G. 1 year after acute Zika virus infection in men. Lancet Infect. Dis. 2020, 20, 25-26. [CrossRef]

18. Arsuaga, M.; Bujalance, S.G.; Díaz-Menéndez, M.; Vázquez, A.; Arribas, J.R. Probable sexual transmission of Zika virus from a vasectomised man. Lancet Infect. Dis. 2016, 16, 1107. [CrossRef]

19. Le Tortorec, A.; Matusali, G.; Mahé, D.; Aubry, F.; Mazaud-Guittot, S.; Houzet, L.; Dejucq-Rainsford, N. From ancient to emerging infections: The odyssey of viruses in the male genital tract. Physiol. Rev. 2020, 100, 1349-1414. [CrossRef]

20. Govero, J.; Esakky, P.; Scheaffer, S.M.; Fernandez, E.; Drury, A.; Platt, D.J.; Gorman, M.J.; Richner, J.M.; Caine, E.A.; Salazar, V.; et al. Zika virus infection damages the testes in mice. Nature 2016, 540, 438-442. [CrossRef]

21. Ma, W.; Li, S.; Ma, S.; Jia, L.; Zhang, F.; Zhang, Y.; Zhang, J.; Wong, G.; Zhang, S.; Lu, X.; et al. Zika Virus Causes Testis Damage and Leads to Male Infertility in Mice. Cell 2016, 167, 1511-1524.e10. [CrossRef]

22. Osuna, C.E.; Lim, S.Y.; Deleage, C.; Griffin, B.D.; Stein, D.; Schroeder, L.T.; Omange, R.; Best, K.; Luo, M.; Hraber, P.T.; et al. Zika viral dynamics and shedding in rhesus and cynomolgus macaques. Nat. Med. 2016, 22, 1448-1455. [CrossRef]

23. Hirsch, A.J.; Smith, J.L.; Haese, N.N.; Broeckel, R.M.; Parkins, C.J.; Kreklywich, C.; DeFilippis, V.R.; Denton, M.; Smith, P.P.; Messer, W.B.; et al. Zika Virus infection of rhesus macaques leads to viral persistence in multiple tissues. PLoS Pathog. 2017, 13, e1006219. [CrossRef]

24. Mansuy, J.M.; Suberbielle, E.; Chapuy-Regaud, S.; Mengelle, C.; Bujan, L.; Marchou, B.; Delobel, P.; Gonzalez-Dunia, D.; Malnou, C.E.; Izopet, J.; et al. Zika virus in semen and spermatozoa. Lancet Infect. Dis. 2016, 16, 1106-1107. [CrossRef]

25. Joguet, G.; Mansuy, J.M.; Matusali, G.; Hamdi, S.; Walschaerts, M.; Pavili, L.; Guyomard, S.; Prisant, N.; Lamarre, P.; DejucqRainsford, N.; et al. Effect of acute Zika virus infection on sperm and virus clearance in body fluids: A prospective observational study. Lancet Infect. Dis. 2017, 17, 1200-1208. [CrossRef]

26. Paz-Bailey, G.; Rosenberg, E.S.; Doyle, K. Persistence of Zika Virus in Body Fluids_Preliminary Report. N. Engl. J. Med. 2018, 379, 1234. [CrossRef]

27. Salam, A.P.; Horby, P.W. The Breadth of Viruses in Human Semen. Emerg. Infect. Dis. 2017, 23, 1922-1924. [CrossRef]

28. Zhao, S.; Zhu, W.; Xue, S.; Han, D. Testicular defense systems: Immune privilege and innate immunity. Cell. Mol. Immunol. 2014, 11, 428-437. [CrossRef]

29. Griswold, M.D. The central role of Sertoli cells in spermatogenesis. Semin. Cell Dev. Biol. 1998, 9, 411-416. [CrossRef]

30. Griswold, M.D. 50 years of spermatogenesis: Sertoli cells and their interactions with germ cells. Biol. Reprod. 2018, 99, 87-100. [CrossRef]

31. França, L.R.; Hess, R.A.; Dufour, J.M.; Hofmann, M.C.; Griswold, M.D. The Sertoli cell: One hundred fifty years of beauty and plasticity. Andrology 2016, 4, 189-212. [CrossRef]

32. Kaur, G.; Thompson, L.A.; Dufour, J.M. Sertoli cells-Immunological sentinels of spermatogenesis. In Seminars in Cell $\mathcal{E}$ Developmental Biology; Academic Press: Cambridge, MS, USA, 2014; p. 36. [CrossRef]

33. Chen, H.; Ge, R.S.; Zirkin, B.R. Leydig cells: From stem cells to aging. Mol. Cell. Endocrinol. 2009, 306, 9-16. [CrossRef]

34. Sheng, Z.Y.; Gao, N.; Wang, Z.Y.; Cui, X.Y.; Zhou, D.S.; Fan, D.Y.; Chen, H.; Wang, P.G.; An, J. Sertoli cells are susceptible to ZIKV infection in mouse testis. Front. Cell. Infect. Microbiol. 2017, 7, 272. [CrossRef] [PubMed] 
35. Strange, D.P.; Green, R.; Siemann, D.N.; Gale, M.; Verma, S. Immunoprofiles of human Sertoli cells infected with Zika virus reveals unique insights into host-pathogen crosstalk. Sci. Rep. 2018, 8, 1-15. [CrossRef] [PubMed]

36. Rashid, M.U.; Zahedi-Amiri, A.; Glover, K.K.; Gao, A.; Nickol, M.E.; Kindrachuk, J.; Wilkins, J.A.; Coombs, K.M. Zika virus dysregulates human sertoli cell proteins involved in spermatogenesis with little effect on tight junctions. PLoS Negl. Trop. Dis. 2020, 14, 1-23. [CrossRef] [PubMed]

37. Kumar, A.; Jovel, J.; Lopez-Orozco, J.; Limonta, D.; Airo, A.M.; Hou, S.; Stryapunina, I.; Fibke, C.; Moore, R.B.; Hobman, T.C. Human sertoli cells support high levels of zika virus replication and persistence. Sci. Rep. 2018, 8, 1-11. [CrossRef] [PubMed]

38. Sielaff, M.; Kuharev, J.; Bohn, T.; Hahlbrock, J.; Bopp, T.; Tenzer, S.; Distler, U. Evaluation of FASP, SP3, and iST Protocols for Proteomic Sample Preparation in the Low Microgram Range. J. Proteome Res. 2017, 16, 4060-4072. [CrossRef] [PubMed]

39. Glover, K.K.M.; Zahedi-Amiri, A.; Lao, Y.; Spicer, V.; Klonisch, T.; Coombs, K.M. Zika Infection Disrupts Proteins Involved in the Neurosensory System. Front. Cell Dev. Biol. 2020, 8, 571. [CrossRef] [PubMed]

40. Kelstrup, C.D.; Bekker-Jensen, D.B.; Arrey, T.N.; Hogrebe, A.; Harder, A.; Olsen, J.V. Performance Evaluation of the Q Exactive HF-X for Shotgun Proteomics. J. Proteome Res. 2018, 17, 727-738. [CrossRef]

41. Coombs, K.M.; Berard, A.; Xu, W.; Krokhin, O.; Meng, X.; Cortens, J.P.; Kobasa, D.; Wilkins, J.; Brown, E.G. Quantitative Proteomic Analyses of Influenza virus-Infected Cultured Human Lung Cells. J. Virol. 2010, 84, 10888-10906. [CrossRef]

42. Candia, J.; Cheung, F.; Kotliarov, Y.; Fantoni, G.; Sellers, B.; Griesman, T.; Huang, J.; Stuccio, S.; Zingone, A.; Ryan, B.M.; et al. Assessment of Variability in the SOMAscan Assay. Sci. Rep. 2017, 7, 1-13. [CrossRef]

43. Siemann, D.N.; Strange, D.P.; Maharaj, P.N.; Shi, P.-Y.; Verma, S. Zika Virus Infects Human Sertoli Cells and Modulates the Integrity of the In Vitro Blood-Testis Barrier Model. J. Virol. 2017, 91, 22. [CrossRef]

44. Jansen, S.; van der Werf, I.M.; Innes, A.M.; Afenjar, A.; Agrawal, P.B.; Anderson, I.J.; Atwal, P.S.; van Binsbergen, E.; van den Boogaard, M.J.; Castiglia, L.; et al. De novo variants in FBXO11 cause a syndromic form of intellectual disability with behavioral problems and dysmorphisms. Eur. J. Hum. Genet. 2019, 27, 20. [CrossRef]

45. Gregor, A.; Sadleir, L.G.; Asadollahi, R.; Azzarello-Burri, S.; Battaglia, A.; Ousager, L.B.; Boonsawat, P.; Bruel, A.L.; Buchert, R.; Calpena, E.; et al. De Novo Variants in the F-Box Protein FBXO11 in 20 Individuals with a Variable Neurodevelopmental Disorder. Am. J. Hum. Genet. 2018, 103, 305-316. [CrossRef]

46. Nemani, T.; Steel, D.; Kaliakatsos, M.; DeVile, C.; Ververi, A.; Scott, R.; Getov, S.; Sudhakar, S.; Male, A.; Mankad, K.; et al. KIF1A-related disorders in children: A wide spectrum of central and peripheral nervous system involvement. J. Peripher. Nerv. Syst. 2020, 25, 117-124. [CrossRef]

47. Mori, T.; Ikeda, D.D.; Yamaguchi, Y.; Unoki, M. NIRF/UHRF2 occupies a central position in the cell cycle network and allows coupling with the epigenetic landscape. FEBS Lett. 2012, 586, 1570-1583. [CrossRef]

48. Wilde, S.; Timpson, A.; Kirsanow, K.; Kaiser, E.; Kayser, M.; Unterländer, M.; Hollfelder, N.; Potekhina, I.D.; Schier, W.; Thomas, M.G.; et al. Direct evidence for positive selection of skin, hair, and eye pigmentation in Europeans during the last 5000 y. Proc. Natl. Acad. Sci. USA 2014, 111, 4832-4837. [CrossRef]

49. Ghodsinejad Kalahroudi, V.; Kamalidehghan, B.; Arasteh Kani, A.; Aryani, O.; Tondar, M.; Ahmadipour, F.; Chung, L.Y.; Houshmand, M. Two Novel Tyrosinase (TYR) Gene Mutations with Pathogenic Impact on Oculocutaneous Albinism Type 1 (OCA1). PLoS ONE 2014, 9, 106656. [CrossRef]

50. Spritz, R.A.; Oh, J.; Fukai, K.; Holmes, S.A.; Ho, L.; Chitayat, D.; France, T.D.; Musarella, M.A.; Orlow, S.J.; Schnur, R.E.; et al. Novel Mutations of the Tyrosinase (TYR) Gene in Type I Oculocutaneous Albinism (OCA1). Hum. Mutat. 1997, 10, 171-174. Available online: https:/ / www.proquest.com/openview/0754b19d664ffd0592b6b7b688100ca7/1?pq-origsite=gscholar\&cbl= 30498 (accessed on 1 January 2022). [CrossRef]

51. Kaneda, H.; Arao, T.; Tanaka, K.; Tamura, D.; Aomatsu, K.; Kudo, K.; Sakai, K.; De Velasco, M.A.; Matsumoto, K.; Fujita, Y.; et al. FOXQ1 is overexpressed in colorectal cancer and enhances tumorigenicity and tumor growth. Cancer Res. 2010, 70, $2053-2063$. [CrossRef]

52. Gao, M.; Shih, I.M.; Wang, T.L. The role of forkhead box Q1 transcription factor in ovarian epithelial carcinomas. Int. J. Mol. Sci. 2012, 13, 13881-13893. [CrossRef]

53. Sun, H.T.; Cheng, S.X.; Tu, Y.; Li, X.H.; Zhang, S. FoxQ1 Promotes Glioma Cells Proliferation and Migration by Regulating NRXN3 Expression. PLoS ONE 2013, 8, e55693. [CrossRef]

54. Xia, L.; Huang, W.; Tian, D.; Zhang, L.; Qi, X.; Chen, Z.; Shang, X.; Nie, Y.; Wu, K. Forkhead box Q1 promotes hepatocellular carcinoma metastasis by transactivating ZEB2 and VersicanV1 expression. Hepatology 2014, 59, 958-973. [CrossRef] [PubMed]

55. Tang, H.; Zheng, J.; Bai, X.; Yue, K.L.; Liang, J.H.; Li, D.Y.; Wang, L.P.; Wang, J.L.; Guo, Q. Forkhead Box Q1 Is Critical to Angiogenesis and Macrophage Recruitment of Colorectal Cancer. Front. Oncol. 2020, 10, 2561. [CrossRef] [PubMed]

56. Christensen, J.; Bentz, S.; Sengstag, T.; Shastri, V.P.; Anderle, P. FOXQ1, a novel target of the Wnt pathway and a new marker for activation of Wnt signaling in solid tumors. PLOS ONE 2013, 8, 3. [CrossRef] [PubMed]

57. Mruk, D.D.; Cheng, C.Y. The Mammalian Blood-Testis Barrier: Its Biology and Regulation. Endocr. Rev. 2015, 36, 564-591. [CrossRef] [PubMed]

58. Vastag, L.; Koyuncu, E.; Grady, S.L.; Shenk, T.E.; Rabinowitz, J.D. Divergent effects of human cytomegalovirus and herpes simplex virus-1 on cellular metabolism. PLoS Pathog. 2011, 7, e1002124. [CrossRef] [PubMed]

59. Thaker, S.K.; Ch'ng, J.; Christofk, H.R. Viral hijacking of cellular metabolism. BMC Biol. 2019, 17, 1-15. [CrossRef] [PubMed] 
60. Fisher, T.N.; Ginsberg, H.S. Accumulation of organic acids by HeLa cells infected with type 4 adenovirus. Proc. Soc. Exp. Biol. Med. 1957, 95, 47-51. [CrossRef]

61. Lewis, V.J.; Scott, L.V. Nutritional requirements for the production of herpes simplex virus. I. Influence of glucose and glutamine of herpes simplex virus production by HeLa cells. J. Bacteriol. 1962, 83, 475-482. [CrossRef]

62. Fontaine, K.A.; Sanchez, E.L.; Camarda, R.; Lagunoff, M. Dengue virus induces and requires glycolysis for optimal replication. J. Virol. 2015, 89, 2358-2366. [CrossRef]

63. Reslan, A.; Haddad, J.G.; Koundi, L.M.; Desprès, P.; Bascands, J.L.; Gadea, G. Zika Virus Growth in Human Kidney Cells Is Restricted by an Elevated Glucose Level. Int. J. Mol. Sci. 2021, 22, 1-13. [CrossRef]

64. Thaker, S.K.; Chapa, T.; Garcia, G., Jr.; Gong, D.; Schmid, E.W.; Arumugaswami, V.; Sun, R.; Christofk, H.R. Differential Metabolic Reprogramming by Zika Virus Promotes Cell Death in Human versus Mosquito Cells. Cell Metab. 2019, 29, 1206-1216. [CrossRef] [PubMed]

65. Jutte, N.H.P.M.; Eikvar, L.; Levy, F.O.; Hansson, V. Metabolism of palmitate in cultured rat Sertoli cells. Reproduction 1985, 73, 497-503. [CrossRef] [PubMed]

66. Li, H.; He, C.; Feng, J.; Zhang, Y.; Tang, Q.; Bian, Z.; Bai, X.; Zhou, H.; Jiang, H.; Heximer, S.P.; et al. Regulator of G protein signaling 5 protects against cardiac hypertrophy and fibrosis during biomechanical stress of pressure overload. Proc. Natl. Acad. Sci. USA 2010, 107, 13818-13823. [CrossRef] [PubMed]

67. Aletti, M.; Lecoules, S.; Kanczuga, V.; Soler, C.; Maquart, M.; Simon, F.; Leparc-Goffart, I. Transient myocarditis associated with acute Zika virus infection. Clin. Infect. Dis. 2017, 64, 678-679. [CrossRef] [PubMed]

68. Minhas, A.M.; Nayab, A.; Iyer, S.; Narmeen, M.; Fatima, K.; Khan, M.S.; Constantin, J. Association of Zika Virus with Myocarditis, Heart Failure, and Arrhythmias: A Literature Review. Cureus 2017, 9. [CrossRef]

69. Li, X.F.; Dong, H.L.; Huang, X.Y.; Qiu, Y.F.; Wang, H.J.; Deng, Y.Q.; Zhang, N.N.; Ye, Q.; Zhao, H.; Liu, Z.Y.; et al. Characterization of a 2016 Clinical Isolate of Zika Virus in Non-human Primates. EBioMedicine 2016, 12, 170. [CrossRef]

70. Strange, D.P.; Jiyarom, B.; Sadri-Ardekani, H.; Cazares, L.H.; Kenny, T.A.; Ward, M.D.; Verma, S. Paracrine IFN Response Limits ZIKV Infection in Human Sertoli Cells. Front. Microbiol. 2021, 12, 1044. [CrossRef]

71. Nie, Y.; Hui, L.; Guo, M.; Yang, W.; Huang, R.; Chen, J.; Wen, X.; Zhao, M.; Wu, Y. Rearrangement of Actin Cytoskeleton by Zika Virus Infection Facilitates Blood-Testis Barrier Hyperpermeability. Virol. Sin. 2021, 36, 692-705. [CrossRef] 